\title{
Aus ,Erfahrung“" wird man selbstwirksam, motiviert und klug: Wie hängen unterschiedliche Komponenten professioneller Kompetenz von Lehramtsstudierenden mit der Nutzung von Lerngelegenheiten zusammen?
}

\author{
Denise Depping (D) Timo Ehmke - Michael Besser
}

Eingegangen: 19. März 2020 / Überarbeitet: 25. August 2020 / Angenommen: 18. Januar 2021 / Online publiziert: 5. Februar 2021

(C) Der/die Autor(en) 2021

Zusammenfassung Im Fokus dieses Beitrags steht die Frage, inwiefern die Nutzung von Lerngelegenheiten (opportunities to learn, OTL) mit Aspekten professioneller Kompetenz von Lehramtsstudierenden zusammenhängt. In einer Querschnittstichprobe von $N=200$ Lehramtsstudierenden wurden das pädagogische Unterrichtswissen, die professionelle Unterrichtswahrnehmung, das pädagogische Interesse und die unterrichtsbezogene Selbstwirksamkeitserwartung erhoben. OTL wurden durch Angaben zur Nutzung universitärer Lehrveranstaltungen, absolvierter Praxisphasen und außeruniversitärer Erfahrungen im Unterrichten erfasst. Die Ergebnisse weisen darauf hin, dass die Nutzung von universitären OTL zur Erklärung des pädagogischen Unterrichtswissens beiträgt; pädagogische Erfahrungen außerhalb der Universität hängen eng mit dem pädagogischen Interesse der Lehramtsstudierenden zusammen. Außerdem mediiert die Selbstwirksamkeitserwartung Zusammenhänge zwischen den erhobenen OTL und den untersuchten Aspekten professioneller Kompetenz. Die Ergebnisse werden im Hinblick auf Gestaltungsmöglichkeiten der Lehramtsausbildung zur Stärkung der Selbstwirksamkeitserwartung und weiterer Aspekte professioneller Kompetenz diskutiert.

Denise Depping arbeitet mittlerweile am Institut für Bildungsmonitoring und Qualitätsentwicklung (IfBQ) in Hamburg. Die Vorarbeiten für das Manuskript sind jedoch noch an der Leuphana Universität entstanden.

D. Depping $(\bowtie) \cdot$ Prof. Dr. T. Ehmke · Prof. Dr. M. Besser

Leuphana Universität Lüneburg, Universitätsallee 1, 21335 Lüneburg, Deutschland

E-Mail: denise.depping@ifbq.hamburg.de

Prof. Dr. T. Ehmke

E-Mail: timo.ehmke@uni.leuphana.de

Prof. Dr. M. Besser

E-Mail: michael.besser@uni.leuphana.de 
Schlüsselwörter Lehramtsausbildung · Professionelle Kompetenz ·

Lerngelegenheiten

\title{
One learns by experience-and becomes self-efficacious as well as motivated. How do different components of student teachers' professional competence relate to the use of learning opportunities?
}

\begin{abstract}
This article focuses on the question to what extent the use of opportunities to learn (OTL) relates to aspects of student teachers' professional competence. In a cross-sectional sample of $N=200$ student teachers, the pedagogical knowledge, the professional perception of teaching, the pedagogical interest and self-efficacy beliefs related to teaching were investigated. OTL were assessed using information on the use of university courses, completed practicum phases and non-university experience in teaching. The results indicate that the use of university OTL contributes to the pedagogical knowledge; pedagogical experience outside the university is closely related to student teachers' pedagogical interest. In addition, self-efficacy beliefs mediate relations between OTL and the examined aspects of professional competence. The results are discussed with regard to the design of teacher training in order to strengthen self-efficacy beliefs and additional aspects of professional competence.
\end{abstract}

Keywords Teacher training Professional competence $\cdot$ Opportunities to learn

\section{Einleitung: Hinführung zum Thema}

Die Lehrperson zählt zu den wichtigsten Einflussfaktoren für den Lernerfolg von Schüler*innen (vgl. Baumert et al. 2010; Hattie 2010). Dabei hat die professionelle Kompetenz (z.B. das pädagogische Unterrichtswissen und die Selbstwirksamkeitserwartung) von Lehrkräften einen nicht zu unterschätzenden Einfluss auf das Lernen von Schüler*innen (Hattie 2010; Tschannen-Moran et al. 1998). Darling-Hammond (vgl. 2000a) zeigt in ihrer Metaanalyse, dass voll ausgebildete Lehrpersonen besser in der Lage sind, Lernprozesse bei Schüler*innen anzustoßen, als Lehrkräfte, die keine oder nur eine unvollständige Ausbildung absolviert haben. Der Effekt der Lehrerausbildung auf die Leistung von Lernenden bleibt sogar unter Kontrolle des sozioökonomischen Status der Lernenden erhalten (Darling-Hammond 2000a). In Anlehnung an das Modell von Baumert und Kunter (vgl. 2006) setzt sich die professionelle Handlungskompetenz von Lehrkräften zusammen aus: 1) Überzeugungen/Werthaltungen, 2) motivationalen Orientierungen (z. B. pädagogisches Interesse, Selbstwirksamkeitserwartung), 3) selbstregulativen Fähigkeiten und 4) dem Professionswissen. Das Professionswissen lässt sich wiederum in Fachwissen (content knowledge, CK), fachdidaktisches Wissen (pedagogical content knowledge, PCK) und (allgemeines) pädagogisches Wissen unterteilen (general pedagogical knowledge, GPK bzw. pedagogical knowledge, PK) (Baumert und Kunter 2006, S. 482; Shulman 1987). Analog zu Angebots-Nutzungs-Modellen im schulischen Kontext (vgl. Helmke 2010) wird auch für die Lehramtsausbildung angenommen, dass durch die Nutzung von Lernangeboten die Kompetenzentwicklung angehender Lehrkräf- 
te unterstützt wird (Blömeke 2013; Terhart et al. 2012, S. 97). Die Entwicklung der einzelnen Aspekte professioneller Kompetenz beginnt idealerweise bereits im Verlauf der universitären Lehramtsausbildung und setzt sich über die gesamte Berufslaufbahn fort. Für den Erwerb von pädagogischem Wissen wird angenommen, dass dieses sich insbesondere im Lehramtsstudium durch die Nutzung von Lerngelegenheiten entwickelt (Terhart et al. 2012; Voss et al. 2015). Inwieweit die Nutzung von Lerngelegenheiten innerhalb und außerhalb eines universitären Lehramtsstudiums mit der Ausprägung unterschiedlicher Facetten der professionellen Kompetenz zusammenhängt, ist bislang noch nicht detailliert erforscht worden. Clark und Newberry (vgl. 2018) merken zum Beispiel an, dass Effekte der Lehramtsausbildung auf die Selbstwirksamkeit bislang nur unzureichend untersucht wurden. Außerdem ist unklar, inwieweit motivationale Orientierungen mit der Nutzung von Lerngelegenheiten zusammenhängen und durch diese verändert werden können (König et al. 2016). Darge und Kolleg*innen (Darge et al. 2012) stellen fest, dass die Wirksamkeit praktischer Lerngelegenheiten näher erforscht werden sollte, um Erkenntnisse über die Gestaltung von Praxisanteilen zu gewinnen. Letztlich führen Tachtsoglou und Kolleg*innen als Forschungsdesiderat an, dass neben Effekten formeller Lerngelegenheiten auch Effekte informeller Lerngelegenheiten auf das pädagogische Wissen untersucht werden sollten (Tachtsoglou und König 2018). Die vorliegende Untersuchung soll einen Beitrag zur Schließung der genannten Forschungslücken leisten, indem Zusammenhänge zwischen formellen und informellen Lerngelegenheiten - theoriebasierte und praxisorientierte - und unterschiedlichen Aspekten professioneller Kompetenz untersucht werden.

Im Folgenden werden zunächst Facetten professioneller Handlungskompetenz von Lehrkräften mit dem Fokus auf pädagogischem Wissen und motivationalen Orientierungen betrachtet. Gegenstand des zweiten Abschnitts sind unterschiedliche Lerngelegenheiten, die zur Entwicklung professioneller Handlungskompetenz beitragen.

\section{Facetten professioneller Handlungskompetenz}

\subsection{Pädagogisches Wissen}

Das Modell professioneller Handlungskompetenz von Lehrkräften (Baumert und Kunter 2006) beschreibt unter anderem das (allgemeine) pädagogische Wissen als einen zentralen Bestandteil der professionellen Kompetenz von Lehrkräften, der die Grundlage für effizienten Unterricht bildet (vgl. Shulman 1987; Voss et al. 2014). Im Gegensatz zum Fachwissen und fachdidaktischen Wissen umfasst PK fachunabhängige Inhalte, die sich zum Beispiel auf das Klassenmanagement beziehen (Shulman 1987). Es wird davon ausgegangen, dass vor allem im universitären Teil der Lehramtsausbildung diese relevante Wissensbasis für wirkungsvolles Handeln aufgebaut wird (Kunina-Habenicht et al. 2013; Terhart et al. 2012). Eine eindeutige Definition des PK und eine klare Abgrenzung zu anderen Begriffen des fächerübergreifenden professionellen Wissens (z.B. bildungswissenschaftliches Wissen) existieren zum jetzigen Zeitpunkt jedoch noch nicht. Voss et al. (vgl. 2015) geben eine Übersicht 
über unterschiedliche Definitionen und Subfacetten von PK sowie Instrumente zu dessen Erhebung. Teilweise werden Überzeugungen und auch die Performanz unter das Konstrukt des pädagogischen Wissens subsumiert. In den meisten Studien bezieht sich PK jedoch ausschließlich auf das unterrichtsbezogene Wissen.

Das pädagogische Wissen besteht unter anderem aus deklarativen und prozeduralen Wissensanteilen (Felbrich et al. 2008; Voss et al. 2015). Das prozedurale Wissen beschreiben Voss und Kolleg*innen ,„[...] als leistungsbezogene Verhaltensdisposition, die sich unabhängig von der authentischen Performanz im Klassenraum durch beispielsweise powerorientierte Testsituationen (z. B. Video- oder Vignettenaufgaben) erfassen lässt“" (Voss et al. 2015, S. 193). Nach Bromme führt eine Verknüpfung des , ,...] (deklarativen) Wissens über Sachverhalte mit dem (prozeduralen) Wissen über Lösungsschritte [...]“ zu Expertise (Bromme 1992, S. 43). In der vorliegenden Untersuchung werden in Anlehnung an Tachtsoglou und König (vgl. 2018) die Strukturierung von Unterricht, die Klassenführung/Motivierung, der Umgang mit Heterogenität und die Leistungsbeurteilung als Facetten von PK definiert. Es werden sowohl deklarative als auch prozedurale Wissensanteile in den Blick genommen, wobei PK ausschließlich Wissensinhalte umfasst und sich weder auf Überzeugungen noch auf Performanz beziehen.

\subsection{Motivationale Orientierungen}

Eine weitere Facette der professionellen Kompetenz von Lehrkräften bilden motivationale Orientierungen. Zu ihnen zählen unter anderem die Selbstwirksamkeitserwartung und die intrinsische Motivation (Baumert und Kunter 2006). Die Selbstwirksamkeitserwartung wird definiert als ,beliefs in one's capabilities to organize and execute the courses of action required to produce given attainments" (Bandura 1997, S. 3) und spiegelt eine eher kognitive Komponente motivationaler Orientierungen wider. Als Quellen der Selbstwirksamkeit werden in Anlehnung an Bandura (vgl. 1997) 1.) persönliche Erfolgserfahrungen, 2.) stellvertretende Erfahrungen (Modelllernen), 3.) verbale Ermutigung (Feedback) und 4.) physiologische und affektive Erregung genannt (vgl. Tschannen-Moran et al. 1998). Da im Lehramtsstudium relativ wenige Gelegenheiten für eigene Erfolgserfahrungen im Unterrichten existieren, gelten diesbezüglich die stellvertretende Erfahrung in Lehrveranstaltungen und die verbale Ermutigung durch Mentor*innen und Dozierende als wirksame Quellen für den Aufbau von Selbstwirksamkeit (Clark und Newberry 2018).

Die Selbstwirksamkeitserwartung ist eng verbunden mit der intrinsischen Motivation (Krapp und Ryan 2002) bzw. mit dem pädagogischen Interesse (Blüthmann et al. 2011), mit dem pädagogischem Wissen (König et al. 2012b) und professionellen Verhaltensweisen von Lehrkräften (Holzberger et al. 2014). Ein Modell von Schiefele und Urhahne (vgl. 2000) zeigt, dass die Selbstwirksamkeitserwartung mit einem höheren Studieninteresse und einer höheren Leistungsmotivation zusammenhängt. Dadurch verfolgen Studierende stärker das Ziel, erfolgreich im Studium zu sein und erzielen höhere Studienleistungen.

Die Selbstwirksamkeitserwartung verändert sich im Verlauf des Studiums (vgl. Müller 2010). Schulte et al. (vgl. 2008) berichten einen Anstieg der unterrichtsbezogenen Selbstwirksamkeitserwartung. Allerdings schätzen sich besonders Lehramts- 
studierende bereits zu Beginn ihres Studiums als überdurchschnittlich kompetent ein (,,unrealistic optimism“, vgl. Weinstein 1989), weil ihnen das Berufsfeld durch ihre eigene Schullaufbahn bereits vertraut ist. Verfügen Lehramtsstudierende darüber hinaus bereits über pädagogische Vorerfahrungen, so wird die Selbstwirksamkeit noch höher eingeschätzt (Kappler 2013). Pädagogische Vorerfahrungen können als Lerngelegenheiten definiert werden, die in Anlehnung an Bandura (vgl. 1997) eigene Erfolgserfahrungen generieren können. Diese stellen die stärkste Quelle für den Aufbau von Selbstwirksamkeit dar.

Das pädagogische Interesse kann als ,gegenstandszentrierte“ Facette intrinsischer Motivation beschrieben werden (Schiefele et al. 2018). Das berufsbezogene Interesse gilt als zeitlich relativ stabil und steht in einem engen Zusammenhang mit der Anstrengungsbereitschaft und dem Durchhaltevermögen im Beruf sowie dem beruflichen Erfolg (van Iddekinge et al. 2011). In einer Untersuchung von Schiefele und Wild (vgl. 2000) zeigte sich außerdem, dass das Studieninteresse sowohl mit der Leistungsmotivation als auch mit der Zielbindung und letztlich mit dem Studienerfolg (Durchschnittsnote) zusammenhängt. Paulick et al. (2013) konnten zeigen, dass bei Lehramtsstudierenden das pädagogische Interesse positiv mit der Selbstwirksamkeitserwartung und günstigen Lernzielorientierungen (mastery, ability approach) zusammenhängt. In einem Strukturgleichungsmodell zeigte sich außerdem, dass das pädagogische Interesse von Lehrkräften vermittelt über weniger Arbeitsvermeidung und einem stärkeren Wunsch, die eigenen unterrichtlichen Fähigkeiten zu zeigen, zu mehr ganzheitlichem Lernen und einem besseren Unterrichtsmanagement beiträgt.

Zusammenfassend lässt sich festhalten, dass sowohl das pädagogische Interesse als gegenstandszentrierte Facette intrinsischer Motivation als auch die Selbstwirksamkeitserwartung eine wichtige Rolle für die Performanz im Studium und im Beruf zu spielen scheinen. Inwiefern Lerngelegenheiten zur Entwicklung der beschriebenen Aspekte professioneller Kompetenz (PK und motivationalen Orientierungen) beitragen, wird im folgenden Absatz erläutert.

\subsection{Lerngelegenheiten}

Lerngelegenheiten werden überwiegend als institutionelle Ausbildungsangebote beschrieben, durch die die Entwicklung unterschiedlicher Aspekte professioneller Handlungskompetenz unterstützt werden kann (Blömeke et al. 2010). Das Konzept der Lerngelegenheiten ist jedoch nicht nur auf formelle und institutionalisierte Angebote (z.B. in der Universität) beschränkt, sondern umfasst auch informelle Lerngelegenheiten außerhalb von Bildungseinrichtungen (Kunina-Habenicht et al. 2013, S. 4) wie bspw. pädagogische Vorerfahrungen (z. B. Erfahrung in der Nachhilfe). Ferner lassen sich Lerngelegenheiten inhaltlich unterteilen in jene, die eher auf die Vermittlung von theoretischen (deklarativen) Wissensinhalten abzielen, und jene, die eher praxisorientiert (prozedural) sind (vgl. König und Seifert 2012).

Entscheidend ist jedoch nicht die Existenz von Lerngelegenheiten per se, sondern die individuelle Nutzung der Ausbildungsangebote (König und Seifert 2012; Kunter et al. 2011; Tachtsoglou und König 2018). Es sollten demnach vor allem die aus Sicht der Studierenden tatsächlich implementierten Inhalte betrachtet werden (Felbrich et al. 2008, S. 328). Das Angebots-Nutzungs-Modell aus der COACTIV- 


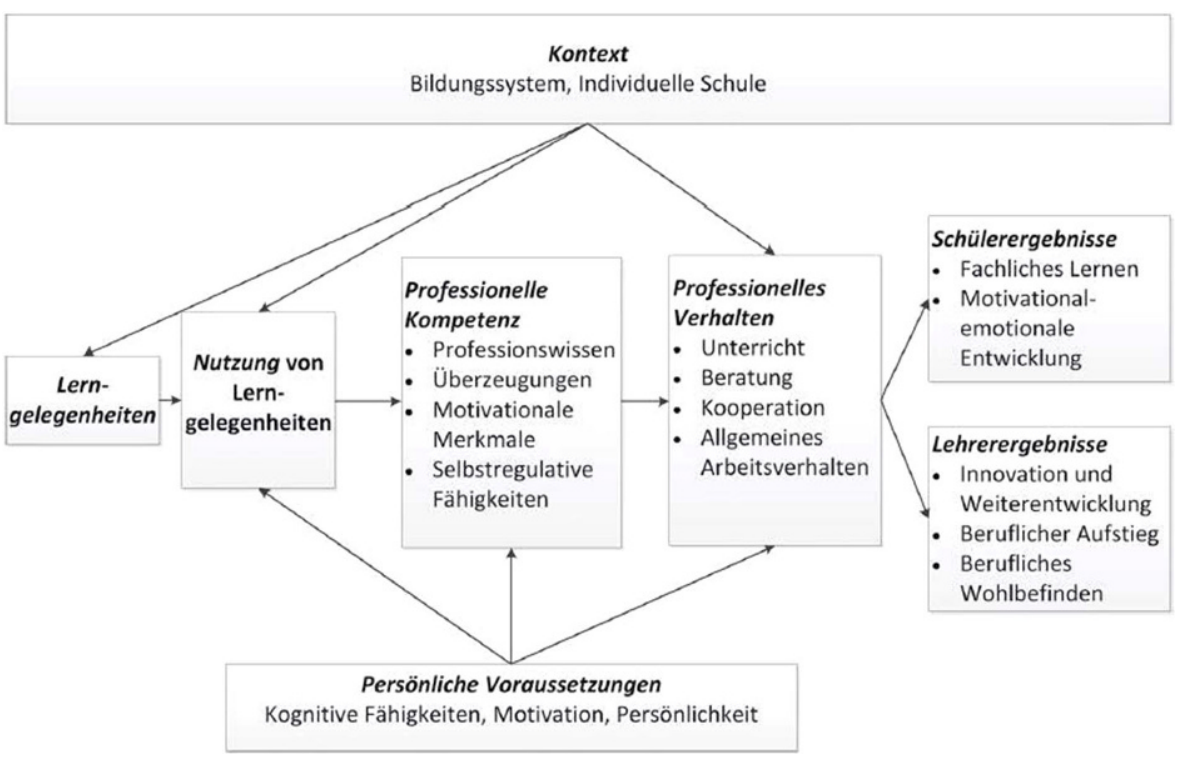

Abb. 1 Angebots-Nutzungs-Modell. (Nach Kunter et al. 2011, S. 59)

Studie (Cognitive Activation in the Classroom, Kunter et al. 2011) verdeutlicht die theoretischen Zusammenhänge zwischen der Nutzung von Lerngelegenheiten und der professionellen Kompetenz von Lehrkräften (vgl. Abb. 1).

Effekte der Nutzung von Lerngelegenheiten im Lehramtsstudium auf das pädagogische Wissen und die Veränderung dieses Wissens wurden unter anderem in der LEK-Studie (König und Seifert 2012), in TEDS-M (Blömeke 2008), in TEDSLT (Blömeke 2013) und in der EMW-Studie (König et al. 2017) gefunden. Auch internationale Studien berichten von positiven Effekten der Nutzung universitärer Lerngelegenheiten auf die Kompetenz von Lehrkräften (Darling-Hammond 2000). Das pädagogische Professionswissen wurde in der TEDS-M- bzw. TEDS-LT- und in der LEK-Studie mit einem Kompetenzmodell gemessen, das die eingesetzten Aufgaben in drei unterschiedliche Bereiche einteilt: 1) Erinnern, 2) Verstehen/Analysieren und 3) Kreieren/Generieren von Handlungsoptionen. Aufgaben im Bereich des Kreierens hingen ausschließlich mit Lerngelegenheiten zusammen, die einen starken Praxisbezug hatten (König und Klemenz 2015, S. 270). Weitere Befunde weisen darauf hin, dass absolvierte Schulpraktika im Rahmen des Lehramtsstudiums einen positiven Einfluss auf das PK haben - insbesondere in Hinblick auf Kenntnisse im Bereich der Didaktik und Pädagogischen Psychologie (König et al. 2012a, S. 261).

Neben Effekten der Nutzung von Lerngelegenheiten auf das Professionswissen existieren auch Studien, die Zusammenhänge zu weiteren Komponenten professioneller Kompetenz untersucht haben. In der Studie von Ronfeldt und Reininger (vgl. 2012) berichten angehende Lehrkräfte nach Praxiserfahrungen über eine höhere Selbstwirksamkeitserwartung im Hinblick auf das Unterrichten. Zudem fühlen sie sich besser auf das Unterrichten vorbereitet. 
Auch von pädagogischen (Vor-) Erfahrungen außerhalb des Studiums wird angenommen, dass sie als Lerngelegenheiten einen positiven Effekt auf den Kompetenzerwerb angehender Lehrer*innen haben (vgl. Schreiber et al. 2012b). In der LEKStudie werden signifikante Korrelationen zwischen der Erfahrung in der Nachhilfe, dem pädagogischen Unterrichtswissen und dem bildungswissenschaftlichen Wissen berichtet (Schreiber et al. 2012b). Darüber hinaus hat sich die Erfahrung in der Nachhilfe als geeigneter Prädiktor für positive Entwicklungen im bildungswissenschaftlichen Wissen erwiesen (König et al. 2012a, S. 272). Doch auch zu anderen Komponenten professioneller Kompetenz werden Beziehungen zu pädagogischen Vorerfahrungen gefunden. Zum Beispiel hängen pädagogische Vorerfahrungen sowohl positiv mit der intrinsischen als auch mit der extrinsischen Berufswahlmotivation zusammen (Lerche et al. 2013; Schreiber et al. 2012b). Im Hinblick auf die Selbstwirksamkeitserwartung finden sich lediglich kleinere positive Zusammenhänge zum wahrgenommenen Erfolg in pädagogischen Vorerfahrungen (Lerche et al. 2013).

Die Berufswahlmotivation und auch die wahrgenommene Fähigkeit, unterrichten zu können, werden durch Lerngelegenheiten - hier im Besonderen durch praxisnahe Lerngelegenheiten - beeinflusst (König et al. 2016).

Da theoretisches Wissen eine wichtige Voraussetzung für kompetentes Handeln darstellt (Schulze-Stocker et al. 2016) und eine hohe Selbstwirksamkeitserwartung mit kompetentem, ausdauerndem und kreativem Handeln einhergeht (TschannenMoran und Hoy 2007), lässt sich vermuten, dass auch eine Verbindung zwischen der Selbstwirksamkeit und dem PK existiert.

Es lässt sich zusammenfassend festhalten, dass die Nutzung von sowohl formellen (Lehrveranstaltungen und Praktika innerhalb des Lehramtsstudiums) als auch informellen Lerngelegenheiten (außeruniversitärer Nachhilfeunterricht) einen Beitrag zum Aufbau unterschiedlicher Facetten professioneller Kompetenz von angehenden Lehrkräften leisten kann. Da insbesondere die Selbstwirksamkeitserwartung bei angehenden Lehrkräften mit mehr Enthusiasmus für den Unterricht und einer effektiveren Unterrichtsführung assoziiert ist (Tschannen-Moran et al. 1998) sowie mit einer höheren Unterrichtsqualität (Holzberger et al. 2013) einhergeht, scheint diesem Konstrukt eine besondere Stellung im Zusammenspiel von Lerngelegenheiten und anderen Aspekten professioneller Kompetenz zuzukommen.

In Rahmen dieser Arbeit sollen die folgenden grundlegenden Forschungsfragen beantwortet werden:

1. Trägt die Nutzung von formellen Lerngelegenheiten im Lehramtsstudium und informellen Lerngelegenheiten außerhalb des Studiums zur Erklärung unterschiedlicher Aspekte professioneller Kompetenz bei Lehramtsstudierenden bei?

2. Ist die Selbstwirksamkeitserwartung ein geeigneter Mediator, der Effekte von der Nutzung formeller und informeller Lerngelegenheiten auf andere Aspekte professioneller Kompetenz bei Lehramtsstudierenden vermittelt?

Zur Untersuchung der Nutzung formeller Lerngelegenheiten wurden die Studierenden einerseits gefragt, wie umfangreich unterschiedliche Lerninhalte in Lehrveranstaltungen thematisiert wurden. Andererseits machten die Studierenden Angaben zu bereits absolvierten Praxisphasen im Studium. Die Nutzung informeller Lerngele- 
genheiten wurde über die Erfahrung im Unterrichten von Kindern und Jugendlichen außerhalb universitärer Praktika operationalisiert.

Außerdem wurden folgende Facetten professioneller Kompetenz von angehenden Lehrkräften erhoben: die Selbstwirksamkeitserwartung im Hinblick auf das Unterrichten, die intrinsische Berufswahlmotivation in Form des pädagogischen Interesses am Lehrberuf, das pädagogische Unterrichtswissen und die professionelle Wahrnehmung von Unterricht. Diese wurde mit einem Videotest operationalisiert, in dem die Studierenden unterrichtsbezogene Qualitätsmerkmale in einer videografierten Unterrichtsstunde identifizieren sollten.

\subsection{Hypothesen}

Auf der Grundlage der berichteten theoretischen Überlegungen und empirischen Befunde werden folgende Hypothesen aufgestellt:

H1 Je intensiver die Nutzung von Lehrveranstaltungen ist, desto

a. mehr pädagogisches Unterrichtswissen ist vorhanden.

b. mehr unterrichtsbezogene Qualitätsaspekte werden in der Videovignette identifiziert.

c. höher ist die Selbstwirksamkeitserwartung im Hinblick auf das Unterrichten.

H2 Je mehr Praxisphasen die Studierenden absolviert haben, desto

a. mehr unterrichtsbezogene Qualitätsaspekte werden in der Videovignette identifiziert.

b. höher ist die Selbstwirksamkeitserwartung im Hinblick auf das Unterrichten.

c. höher ist das pädagogische Interesse am Lehrberuf.

H3 Je größer die Erfahrung im Unterrichten außerhalb des Lehramtsstudiums ist, desto

a. mehr pädagogisches Unterrichtswissen ist vorhanden.

b. mehr unterrichtliche Qualitätsaspekte werden identifiziert.

c. höher ist die Selbstwirksamkeitserwartung im Hinblick auf das Unterrichten.

d. höher ist das pädagogische Interesse am Lehrberuf.

H4 Die Selbstwirksamkeitserwartung mediiert Effekte von der Nutzung universitärer Lehrveranstaltungen auf

a. das pädagogische Unterrichtswissen.

b. die Anzahl identifizierter Qualitätsaspekte in der Videovignette.

H5 Die Selbstwirksamkeitserwartung mediiert Effekte von der Anzahl absolvierter universitärer Praxisphasen auf

a. das pädagogische Unterrichtswissen.

b. die Anzahl identifizierter Qualitätsaspekte in der Videovignette.

c. das pädagogische Interesse am Lehrberuf. 
H6 Die Selbstwirksamkeitserwartung mediiert Effekte von der außeruniversitären Erfahrung im Unterrichten auf

a. das pädagogische Unterrichtswissen.

b. die Anzahl identifizierter Qualitätsaspekte in der Videovignette.

c. das pädagogische Interesse am Lehrberuf.

\section{Methoden}

\subsection{Stichprobe}

Die Daten der Studie stammen aus der LeveL-Studie (Lernentwicklungsverläufe im Lehramtsstudium), die an der Leuphana Universität Lüneburg durchgeführt wurde. Finanziert wurde die Studie mit Mitteln der Telekom-Stiftung (Recruitment - Assessment - Support, Projektleitung Prof. Dr. Timo Ehmke und Prof. Dr. Dominik Leiß). Insgesamt nahmen zu Beginn des Wintersemesters 2015 N=274 Lehramtsstudierende aus unterschiedlichen Fachsemestern des Bachelor- und Masterstudiengangs im Rahmen von Lehrveranstaltungen oder in zusätzlich eingerichteten Testzeiträumen teil. Da an dem Studienstandort kein Studium für das Gymnasiallehramt angeboten wird, bestand die Stichprobe aus Studierenden des Grund-, Haupt- und Realschullehramts und des Lehramts für berufsbildende Schulen. Im Durchschnitt befanden sich die Studierenden zum Zeitpunkt der Befragung im fünften Fachsemester $(\mathrm{M}=4,80, \mathrm{SD}=2,60) .89 \%$ der Studierenden waren weiblich.

\subsection{Lerngelegenheiten}

Die individuelle Nutzung von Lerngelegenheiten wurde mit einem Fragebogen erhoben. Die teilnehmenden Studierenden gaben an, in welchem Ausmaß bestimmte Lerninhalte in den von ihnen besuchten Lehrveranstaltungen bereits thematisiert wurden. Die Lerninhalte waren thematisch eng mit den Fragen im Wissenstest verknüpft (Schulze-Stocker et al. 2016). Da es an der Leuphana Universität keine Anwesenheitspflicht in den Lehrveranstaltungen gibt, stellt die Angabe über das Ausmaß behandelter Themen einen Indikator für die tatsächliche Nutzung der Lerngelegenheiten dar. Außerdem wurde erfragt, wie viele Schulpraktika bereits im Rahmen des Studiums absolviert wurden. An der Leuphana Universität dient das erste Praktikum im zweiten Bachelorsemester (schulpraktische Studien 1, SPS 1) der Berufserkundung und umfasst drei Wochen. Die Studierenden beobachten vorwiegend Unterrichtssituationen und lernen die Institution Schule kennen. Begleitend finden eine Vorlesung und fakultativ ein Tutorium statt. Im vierwöchigen SPS 2 (schulpraktische Studien 2), das im vierten und fünften Bachelorsemester stattfindet, führen die Studierenden bereits Lernarrangements unter Anleitung durch und reflektieren unterrichtliche Prozesse im Hinblick auf Didaktik und Methodik. Zum SPS 2 werden ein Begleitseminar und fakultativ ein Tutorium angeboten. Das dritte Praktikum findet im zweiten Mastersemester statt. Bereits im ersten Mastersemester besuchen die Studierenden ein vorbereitendes Praxismodul. Für jedes ihrer zwei Unterrichtsfächer wird ein Seminar angeboten, in dem sie unter anderem didaktische Fragestellungen 
Tab. 1 Skalen zur Erhebung der Lerngelegenheiten

\begin{tabular}{|c|c|c|c|c|c|}
\hline Skala & Beispiel-Item & $\begin{array}{l}\text { Item- } \\
\text { Anzahl }\end{array}$ & $\begin{array}{l}\text { Minimum/ } \\
\text { Maximum }\end{array}$ & $\begin{array}{l}\mathrm{M} \\
(S D)\end{array}$ & $\alpha$ \\
\hline $\begin{array}{l}\text { Nutzung uni- } \\
\text { versitärer Lern- } \\
\text { gelegenheiten } \\
\text { (LV) }\end{array}$ & $\begin{array}{l}\text { Inwiefern wurden bisher folgende Berei- } \\
\text { che in Ihrer Lehramtsausbildung themati- } \\
\text { siert? } \\
\text { "Analyse von Unterrichtseinheiten“ } \\
{[0=\text { gar nicht }-4=\text { mehrere spezifische }} \\
\text { Lehrveranstaltungen] }\end{array}$ & 9 & $0 . / 3,78$ & $\begin{array}{l}1,70 \\
(0,96)\end{array}$ & 0,91 \\
\hline $\begin{array}{l}\text { Anzahl der } \\
\text { absolvierten } \\
\text { universitären } \\
\text { Praxisanteile } \\
\text { (PA) }\end{array}$ & $\begin{array}{l}{[1=\operatorname{SPS} 1,2=\operatorname{SPS} 1 \text { und SPS } 2,} \\
3=\text { SPS } 1, \text { SPS } 2 \text { und Praxisblock }]\end{array}$ & 1 & $0 / 3$ & $\begin{array}{l}1,33 \\
(0,98)\end{array}$ & - \\
\hline $\begin{array}{l}\text { Außeruniversitäre } \\
\text { Erfahrung im } \\
\text { Unterrichten } \\
\text { (EU) }\end{array}$ & $\begin{array}{l}\text { „Ich habe Erfahrung ... } \\
\text { im Unterrichten von Kindern bzw. Ju- } \\
\text { gendlichen (z. B. Nachhilfe).“ } \\
{[0=\text { gar nicht }-4=\text { mehrere Jahre }]}\end{array}$ & 1 & $0 / 4$ & $\begin{array}{l}2,56 \\
(1,37)\end{array}$ & - \\
\hline
\end{tabular}

erarbeiten und Unterrichtsentwürfe entwickeln. Der Praxisblock umfasst 18 Unterrichtswochen, in denen die Studierenden mindestens 15 Stunden an mindestens drei Tagen pro Woche in der Schule absolvieren. Zusätzlich finden für jedes Unterrichtsfach mindestens drei Beratungstermine statt. Neben der eigenständigen Planung und Durchführung von Unterricht beteiligen sich die Studierenden auch an Prozessen der Schulentwicklung und reflektieren die Anforderungen des Berufsfeldes Schule. Der Praxisblock wird flankiert von je einem Begleit- und Nachbereitungsseminar pro Unterrichtsfach, das der Reflexion von Praxiserfahrungen und der Verknüpfung mit fachdidaktischen Theorien dient. Im Hinblick auf informelle Lerngelegenheiten wurde der Grad der Erfahrung im Unterrichten von Kindern (z. B. Nachhilfe geben) erhoben. Beispiel-Items und psychometrische Eigenschaften für die Skalen zur Erhebung der Lerngelegenheiten sind Tab. $1 \mathrm{zu}$ entnehmen.

\subsection{Aspekte professioneller Kompetenz}

\subsubsection{Pädagogisches Unterrichtswissen}

Zur Erhebung des Professionswissens wurde ein Testinstrument eingesetzt, das schwerpunktmäßig deklaratives pädagogisches Unterrichtswissen misst. Die Items stammen überwiegend aus der TEDS-M-Studie (Blömeke 2008) und der LEK-Studie (König und Seifert 2012). Vereinzelt wurden selbstentwickelte Aufgaben verwendet, die sich thematisch auf spezifische Inhalte der Lehrveranstaltungen des Studienstandorts beziehen. Aufgrund der begrenzten Testzeit wurde ausschließlich das Unterrichtswissen gemessen, da es eine ,zentrale berufliche Aufgabe“ (König 2012, S. 146) von Lehrkräften darstellt. Zur Erhebung des prozeduralen Unterrichtswissens wurde den Lehramtsstudierenden zweimalig eine siebenminütige Videosequenz einer realen Unterrichtsstunde in Mathematik präsentiert. Die Studierenden sollten anschließend positive und negative Qualitätsaspekte der Mathematikstunde auf der Grundlage von Merkmalen „guten Unterrichts“ (Helmke 2006) nennen. Für die 
Auswertung wurden Einschätzungen von erfahrenen Mathematiklehrkräften herangezogen und als Referenzwerte verwendet. Die genannten Qualitätsaspekte wurden anschließend von den Lehrkräften hinsichtlich ihrer Relevanz für effektiven Unterricht gewichtet.

\subsubsection{Intrinsische Berufswahlmotivation}

Als Teilaspekt der intrinsischen Berufswahlmotivation wurden fünf Items zur Untersuchung des pädagogischen Interesses vorgelegt. Zwei Items stammen aus der FEMOLA-Skala (Pohlmann und Möller 2010) und beziehen sich auf die allgemeine Freude an der Arbeit mit Kindern und die Wichtigkeit, einen Beitrag zu ihrer Ausbildung zu leisten. Drei weitere Items wurden ergänzt, die in der EMW-Studie verwendet wurden (Schreiber et al. 2012a) und ihren Ursprung in der deutschen Übersetzung des FIT-Choice-Fragebogens haben. Inhaltlich beziehen sich die Items auf die Vermittlung von Werten, die Aufhebung sozialer Benachteiligung und die Unterstützung beim Lernen. Diese inhaltliche Ausrichtung der Items wurde gewählt, da sich auch viele Items im Wissenstest auf diese Aspekte beziehen.

Tab. 2 Skalen zur Erhebung der professionellen Kompetenz

\begin{tabular}{|c|c|c|c|c|c|}
\hline Skala & Beispiel-Item & $\begin{array}{l}\text { Item- } \\
\text { Anzahl }\end{array}$ & $\begin{array}{l}\text { Minimum/ } \\
\text { Maximum }\end{array}$ & $\begin{array}{l}\mathrm{M} \\
(S D)\end{array}$ & $\alpha$ \\
\hline $\begin{array}{l}\text { Pädagogisches } \\
\text { Unterrichtswis- } \\
\text { sen }(\mathbf{P K})\end{array}$ & $\begin{array}{l}\text { Mit welchen Lehr-Lernformen bzw. } \\
\text { Lernmaterialien kann man Schüler/innen } \\
\text { zum selbstständigen Lernen anregen? } \\
\text { (Bitte antworten Sie in Stichpunkten. } \\
\text { Geben Sie mehrere Methoden an, idealer- } \\
\text { weise drei.) } \\
\text { Welche vier Sozialformen des Schulun- } \\
\text { terrichts gibt es? }\end{array}$ & 18 & $0 / 0,92$ & $\begin{array}{l}0,49 \\
(0,18)\end{array}$ & 0,74 \\
\hline $\begin{array}{l}\text { Professionelle } \\
\text { Wahrnehmung } \\
\text { von Unterricht } \\
\text { (PW) }\end{array}$ & $\begin{array}{l}\text { Wie schätzen Sie die allgemeine Qualität } \\
\text { des Unterrichts in der gezeigten Video- } \\
\text { sequenz ein? Bitte beschreiben Sie (in } \\
\text { Stichpunkten), welche Aspekte Ihnen in } \\
\text { der Unterrichtssituation positiv und wel- } \\
\text { che Aspekte Ihnen negativ aufgefallen } \\
\text { sind }\end{array}$ & 1 & $0,02 / 0,58$ & $\begin{array}{l}0,25 \\
(0,08)\end{array}$ & - \\
\hline $\begin{array}{l}\text { Pädagogisches } \\
\text { Interesse (PI) }\end{array}$ & $\begin{array}{l}\text { „Ich habe das Lehramtsstudium gewählt, } \\
\text { weil ... } \\
\text { ich Kindern und Jugendlichen helfen } \\
\text { möchte zu lernen.“ } \\
\text { [1 = trifft gar nicht } \mathrm{zu}-4=\text { trifft völlig } \\
\mathrm{zu}]\end{array}$ & 5 & $2,20 / 4,00$ & $\begin{array}{l}3,58 \\
(0,38)\end{array}$ & 0,72 \\
\hline $\begin{array}{l}\text { Selbstwirksam- } \\
\text { keitserwartung } \\
\text { (SW) }\end{array}$ & $\begin{array}{l}\text { „Ich traue mir zu, die Schüler/innen für } \\
\text { neue Projekte zu begeistern.“ } \\
{[1=\text { trifft gar nicht zu }-4=\text { trifft völlig }} \\
\text { zu }]\end{array}$ & 9 & $1,78 / 4,00$ & $\begin{array}{l}3,10 \\
(0,40)\end{array}$ & 0,78 \\
\hline
\end{tabular}




\subsubsection{Selbstwirksamkeitserwartung}

Die Selbstwirksamkeitserwartung im Hinblick auf das Unterrichten wurde mit bereits validierten Items (Schulte 2008; Schwarzer und Jerusalem 1999) erhoben und durch Eigenkonstruktionen ergänzt.

Beispielitems und psychometrische Eigenschaften für die Skalen zur Messung von Aspekten professioneller Kompetenz sind Tab. 2 zu entnehmen.

\subsection{Fachsemester}

Das Fachsemester der Lehramtsstudierenden wurde als Kontrollvariable in das Modell eingefügt, um weitere Effekte der Ausbildungszeit zu berücksichtigen.

\subsection{Statistische Auswertung}

Zur Überprüfung der Gütekriterien wurde für die offenen Fragen im Test zum pädagogischen Unterrichtswissen die Interrater-Reliabilität zwischen zwei vorher geschulten Ratern bestimmt. Da die überwiegende Mehrheit der Fragen nicht dichotom ist, wurde der Intra-Class-Koeffizient berechnet. Der ICC erreicht einen Wert von 0,67, was nach (Cicchetti 1994, S. 286) für eine gute Übereinstimmung spricht.

Die angenommenen Zusammenhänge wurden mit einem Strukturgleichungsmodell (structural equation modeling, SEM) überprüft. Zur Berechnung des SEM wurde die Statistiksoftware Mplus (Version 7.3) genutzt. Fehlende Werte wurden mit der Full Information Maximum Likelihood-Methode (FIML) geschätzt. Vor der Berechnung der Pfadmodelle wurden zunächst einzelne Messmodelle für die latenten Variablen berechnet und die Items mit der höchsten Faktorladung auf 1 fixiert. Das pädagogische Unterrichtswissen, das pädagogische Interesse und die Selbstwirksamkeitserwartung gingen als latente Variablen in das Modell ein. Indirekte Effekte im Mediationsmodell wurden mit der multivariaten Delta-Methode auf Signifikanz überprüft (MacKinnon 2008).

Tab. 3 Interkorrelationen zwischen Lerngelegenheiten und Aspekten professioneller Kompetenz

\begin{tabular}{llllllll}
\hline & $\mathrm{LV}$ & $\mathrm{PA}$ & $\mathrm{EU}$ & $\mathrm{SW}$ & $\mathrm{PI}$ & $\mathrm{PK}$ & $\mathrm{PW}$ \\
\hline $\mathrm{LV}$ & 1 & - & - & - & - & - & - \\
$\mathrm{PA}$ & $0,66^{* * *}$ & 1 & - & - & - & - & - \\
$\mathrm{EU}$ & $0,16^{*}$ & $0,17^{* *}$ & 1 & - & - & - & - \\
$\mathrm{SW}$ & $0,43^{* * *}$ & $0,41^{* * *}$ & $0,27 * * *$ & 1 & - & - & - \\
$\mathrm{PI}$ & $0,14^{*}$ & 0,09 & $0,17 *$ & $0,50 * * *$ & 1 & - & - \\
$\mathrm{PK}$ & $0,60^{* * *}$ & $0,66^{* * *}$ & $0,17 *$ & $0,41^{* * *}$ & 0,13 & 1 & - \\
$\mathrm{PW}$ & $0,23 * * *$ & $0,20^{* *}$ & $0,14 * *$ & $0,24 * * *$ & $0,22^{* * *}$ & $0,37 * * *$ & 1 \\
$\mathrm{FS}$ & $0,62^{* * *}$ & $0,83^{* * *}$ & 0,10 & $0,34 * *$ & 0,01 & $0,68 * * *$ & $0,23 * * *$ \\
\hline
\end{tabular}

$\chi^{2}(252)=397,668, \chi^{2} / \mathrm{df}=1,58, p<0,001 ; \mathrm{TLI}=0,91 ; \mathrm{CFI}=0,92 ; \mathrm{RMSEA}=0,05$

$L V$ Nutzung von Lehrveranstaltungen, $P A$ universitäre Praxisanteile, $E U$ außeruniversitäre Erfahrung im Unterrichten, $S W$ Selbstwirksamkeitserwartung, $P I$ Pädagogisches Interesse, $P K$ pädagogisches Unterrichtswissen, $P W$ Professionelle Wahrnehmung von Unterricht, FS Fachsemester $* * * p<0,001, * * p<0,01, * p<0,05,{ }^{\#} p<0,1$ 
Die 18 Items aus dem Wissenstest zum pädagogischen Unterrichtswissen wurden zu sechs Parceln aus jeweils drei Items zusammengefasst. Die zusammengefassten Items wurden hinsichtlich ihrer Schwierigkeit ausbalanciert. Dieses Vorgehen wurde gewählt, um mit einem zuverlässigeren und stabileren Messmodell das dahinterliegende Konstrukt des pädagogischen Unterrichtswissens bestmöglich zu identifizieren (Little et al. 2002).

\section{Ergebnisse}

Im Folgenden werden zunächst die Interkorrelationen zwischen den latenten bzw. manifesten Variablen der Lerngelegenheiten und den Aspekten professioneller Kompetenz berichtet (vgl. Tab. 3). Es zeigt sich, dass die Nutzung von universitären Lehrveranstaltungen sowohl mit dem Unterrichtswissen als auch mit der professionellen Unterrichtswahrnehmung zusammenhängt. Entgegen der Erwartung gibt es keine Zusammenhänge zwischen den absolvierten Praxisphasen und dem pädagogischen Interesse. Diese Facette der intrinsischen Motivation korreliert jedoch positiv mit der Nutzung der Lehrveranstaltungen. Die postulierten Zusammenhänge zwischen der außeruniversitären Erfahrung im Unterrichten mit den untersuchten Aspekten der professionellen Kompetenz werden durch die Ergebnisse bestätigt. Zusätzlich ist die außeruniversitäre Erfahrung im Unterrichten positiv mit dem Unterrichtswissen verknüpft. Alle untersuchten Lerngelegenheiten korrelieren signifikant positiv mit

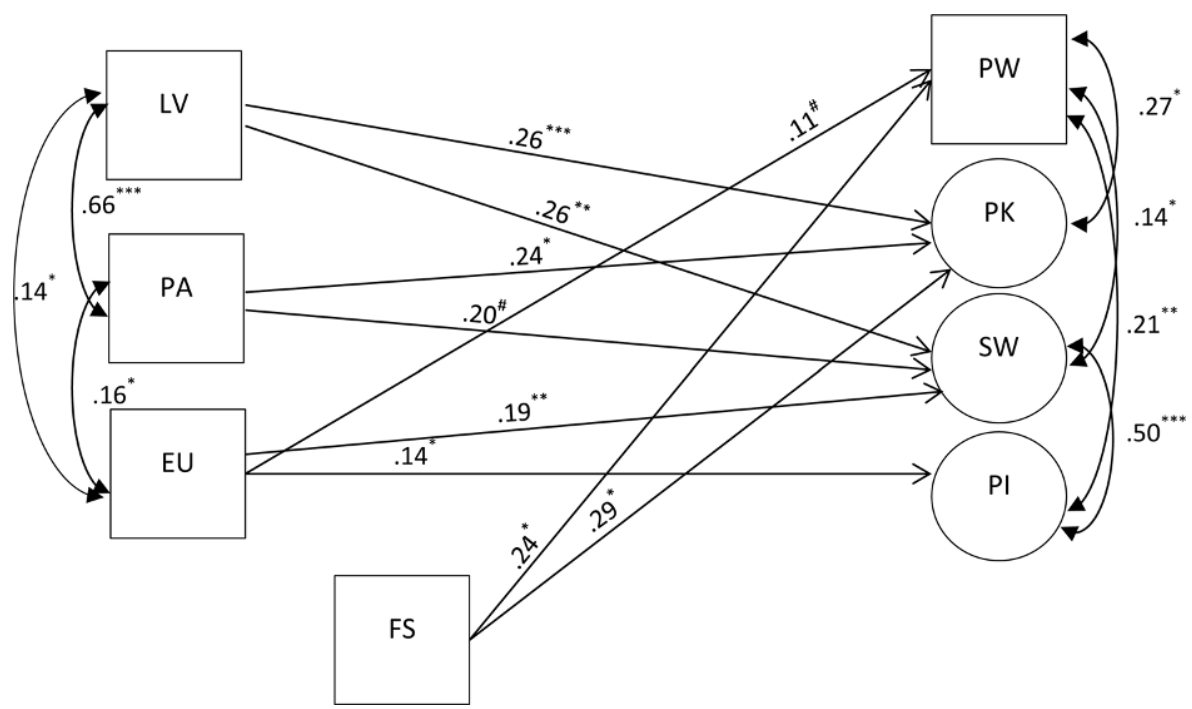

Abb. 2 Strukturgleichungsmodell zur Berechnung von Effekten formeller und informeller Lerngelegenheiten auf Aspekte professioneller Kompetenz. $\left(\chi^{2}(254)=396,572, p<0,001, \chi^{2} / \mathrm{df}=1,56\right.$; TLI=0,88; $\mathrm{CFI}=0,90$; RMSEA = 0,05. $L V$ Nutzung von Lehrveranstaltungen, $P A$ universitäre Praxisanteile, $E U$ außeruniversitäre Erfahrung im Unterrichten, $S W$ Selbstwirksamkeitserwartung, PI Pädagogisches Interesse, $P K$ pädagogisch-psychologisches Unterrichtswissen, $P W$ Professionelle Wahrnehmung von Unterricht, FS Fachsemester. $\left.* * * p<0,001, * * p<0,01,{ }^{*} p<0,05,{ }^{*} p<0,1\right)$ 
der Selbstwirksamkeitserwartung. Darüber hinaus hängt die Selbstwirksamkeitserwartung mit allen untersuchten Facetten der professionellen Kompetenz angehender Lehrkräfte zusammen.

\subsection{Strukturgleichungsmodell}

In Abb. 2 sind die Koeffizienten für das Strukturgleichungsmodell abgebildet. Alle manifesten Items laden signifikant auf die latenten Variablen, daher wird aus Gründen der Übersichtlichkeit ausschließlich das Pfadmodell ohne die Messmodelle gezeigt. In das Modell wurden zu Gunsten der Stabilität nur Pfade zwischen Variablen aufgenommen, die Korrelationen von $p<0,1$ aufweisen. Des Weiteren werden in der Abbildung des SEM nur die Pfade angezeigt, die einen $p$-Wert $<0,1$ aufweisen. Der Modelfit des SEM ohne Mediation liegt im akzeptablen Bereich. Da der $\chi^{2}$-Wert bei größeren Stichproben auch bei praktisch nicht bedeutsamen Abweichungen signifikant wird, wurde dieser in Anlehnung an Kline (vgl. 2016) durch die Freiheitsgrade dividiert. Werte $<3$ sprechen für einen guten Modelfit (vgl. Kline 2016).

Im Folgenden werden die Ergebnisse entlang der Hypothesen H1 bis H3 berichtet. So gilt: Das pädagogische Unterrichtswissen (H1a) und die Ausprägung der Selbstwirksamkeitserwartung (H1c) lassen sich durch die Nutzung der universitären Lehrveranstaltungen erklären $(\beta=0,26, p<0,001$ und $\beta=0,26, p=0,002)$. Jedoch identifizieren Studierende, die mehr universitäre Lehrveranstaltungen zu unterschiedlichen pädagogisch-psychologischen Inhalten besucht haben, nicht signifikant mehr Qualitätsaspekte guten Unterrichts in der Videovignette (H1b) $(\beta=0,12$, $p=0,139)$.

Die Anzahl absolvierter universitärer Praxisphasen trägt ebenfalls nicht zu einer professionelleren Analyse der Videovignette bei (H2a) $(\beta=-0,10, p=0,379)$. $\mathrm{Da}$ es bereits bei den Interkorrelationen keinen signifikanten Zusammenhang mit dem pädagogischen Interesse gab (H2c) $(r=0,09, p=0,183)$, wurde diese Facette der intrinsischen Motivation nicht in das SEM aufgenommen. Die Selbstwirksamkeitserwartung der Studierenden lässt sich nicht durch die Anzahl absolvierter Praxisphasen erklären (H2b) $(\beta=0,20, p=0,077)$. Jedoch stellt die Anzahl absolvierter Praxisanteile einen signifikanten Prädiktor für das pädagogische Unterrichtswissen $\operatorname{dar}(\beta=0,24, p=0,026)$.

Die pädagogische Erfahrung im Unterrichten außerhalb des Lehramtsstudiums (Nachhilfe geben) wird als Prädiktor ausschließlich für das pädagogische Interesse und die Selbstwirksamkeitserwartung signifikant (H3c und $\mathrm{H} 3 \mathrm{~d})(\beta=0,14, p=0,026$ und $\beta=0,19, p=0,002)$. Sie trägt weder zur Erklärung der professionellen Wahrnehmung von Unterricht im Videotest bei (H3b) $(\beta=0,11, p=0,067)$ noch erklärt sie das pädagogische Unterrichtswissen (H3a) $(\beta=0,07, p=0,215)$. Die Hypothesen H1 bis H3 konnten somit nur teilweise durch die Ergebnisse gestützt werden.

Die Varianzaufklärung der unterschiedlichen Aspekte professioneller Kompetenz in dem berechneten Strukturgleichungsmodell fällt mit $53 \%$ für das pädagogische Unterrichtswissen am höchsten aus. Die Selbstwirksamkeitserwartung wird durch die Prädiktoren zu 24\% aufgeklärt. Die erhobenen Lerngelegenheiten besitzen nur eine geringe, aber noch signifikante Vorhersagekraft für den professionellen Blick 


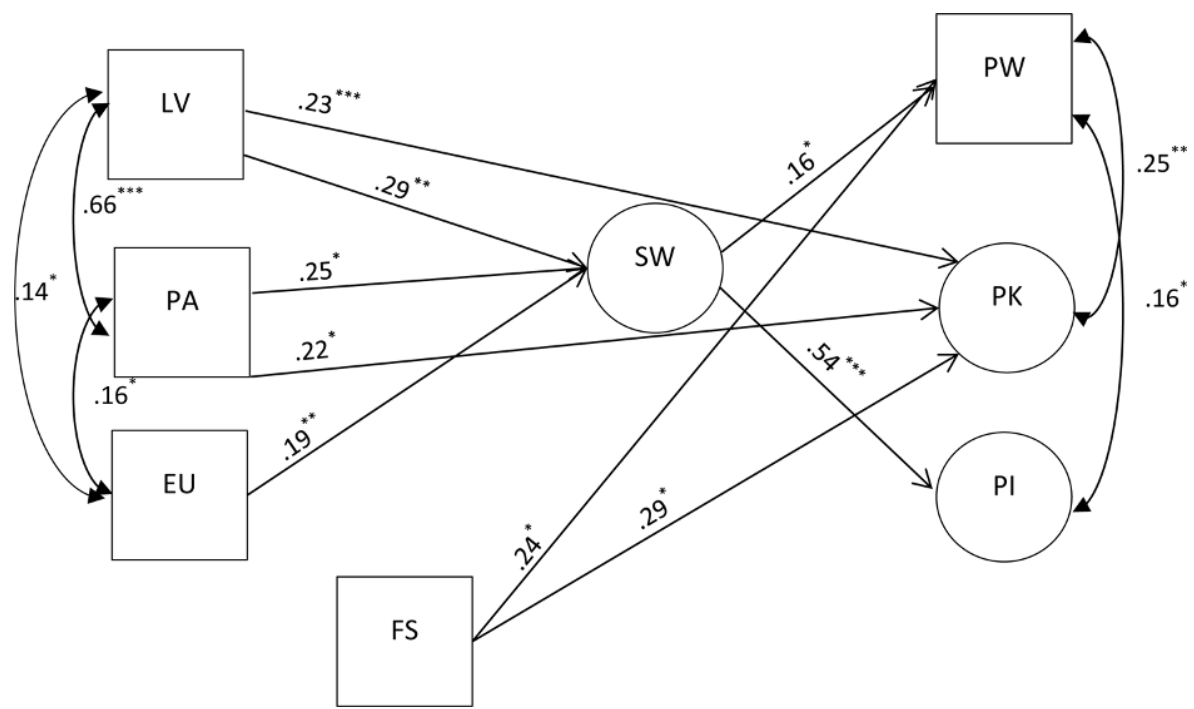

Abb. 3 Strukturgleichungsmodell mit Mediation zur Berechnung von Effekten formeller und informeller Lerngelegenheiten auf Aspekte professioneller Kompetenz. $\left(\chi^{2}(254)=398,519, p<0,001, \chi^{2} / \mathrm{df}=1,57\right.$; $\mathrm{TLI}=0,88$; $\mathrm{CFI}=0,90$; RMSEA $=0,05 . L V$ Nutzung von Lehrveranstaltungen, $P A$ universitäre Praxisanteile, $E U$ außeruniversitäre Erfahrung im Unterrichten, $S W$ Selbstwirksamkeitserwartung, PI Pädagogisches Interesse, $P K$ pädagogisch-psychologisches Unterrichtswissen, $P W$ Professionelle Wahrnehmung von Unterricht, FS Fachsemester. $\left.* * * p<0,001, * * p<0,01, * p<0,05,{ }^{*} p<0,1\right)$

auf Unterricht $\left(R^{2}=8 \%, p=0,013\right)$. Das pädagogische Interesse am Lehrberuf lässt sich durch die Prädiktoren im Modell nicht erklären $\left(R^{2}=4 \%, p=0,17\right)$.

\subsection{Mediationsmodell}

Im Folgenden werden die Ergebnisse für das Mediationsmodell entlang der Hypothesen H4 bis H6 berichtet. Die Modellparameter des Mediationsmodells weisen ähnlich wie bei dem ersten SEM einen akzeptablen Modellfit auf. Abb. 3 zeigt die Koeffizienten des Mediationsmodells.

Der direkte Effekt von der Nutzung der Lehrveranstaltungen auf das pädagogische Unterrichtswissen bleibt bestehen und wird nicht durch die Selbstwirksamkeitserwartung mediiert $(\beta=0,03, p=0,150)(\mathrm{H} 4 \mathrm{a})$. Darüber hinaus trägt eine höhere Selbstwirksamkeitserwartung auch nicht dazu bei, dass Studierende, die in einem größeren Ausmaß Lehrveranstaltungen genutzt haben, mehr unterrichtsbezogene Qualitätsaspekte in der Videovignette identifizieren $(\beta=0,05, p=0,080)(\mathrm{H} 4 \mathrm{~b})$. Entgegen der Erwartung wird das pädagogische Interesse indirekt durch die Nutzung universitärer Lehrveranstaltungen erklärt $(\beta=0,16, p=0,003)$.

Die Anzahl der absolvierten universitären Praxisphasen trägt weiterhin ausschließlich direkt zur Erklärung des Unterrichtswissens bei $(\beta=0,22, p=0,044)$ (H5a). Weder die professionelle Wahrnehmung der videografierten Unterrichtsstunde noch das pädagogische Interesse werden indirekt durch die Selbstwirksamkeitserwartung erklärt (H5b und H5c) $(\beta=0,13, p=0,056$ und $\beta=-0,12, p=0,306)$. 


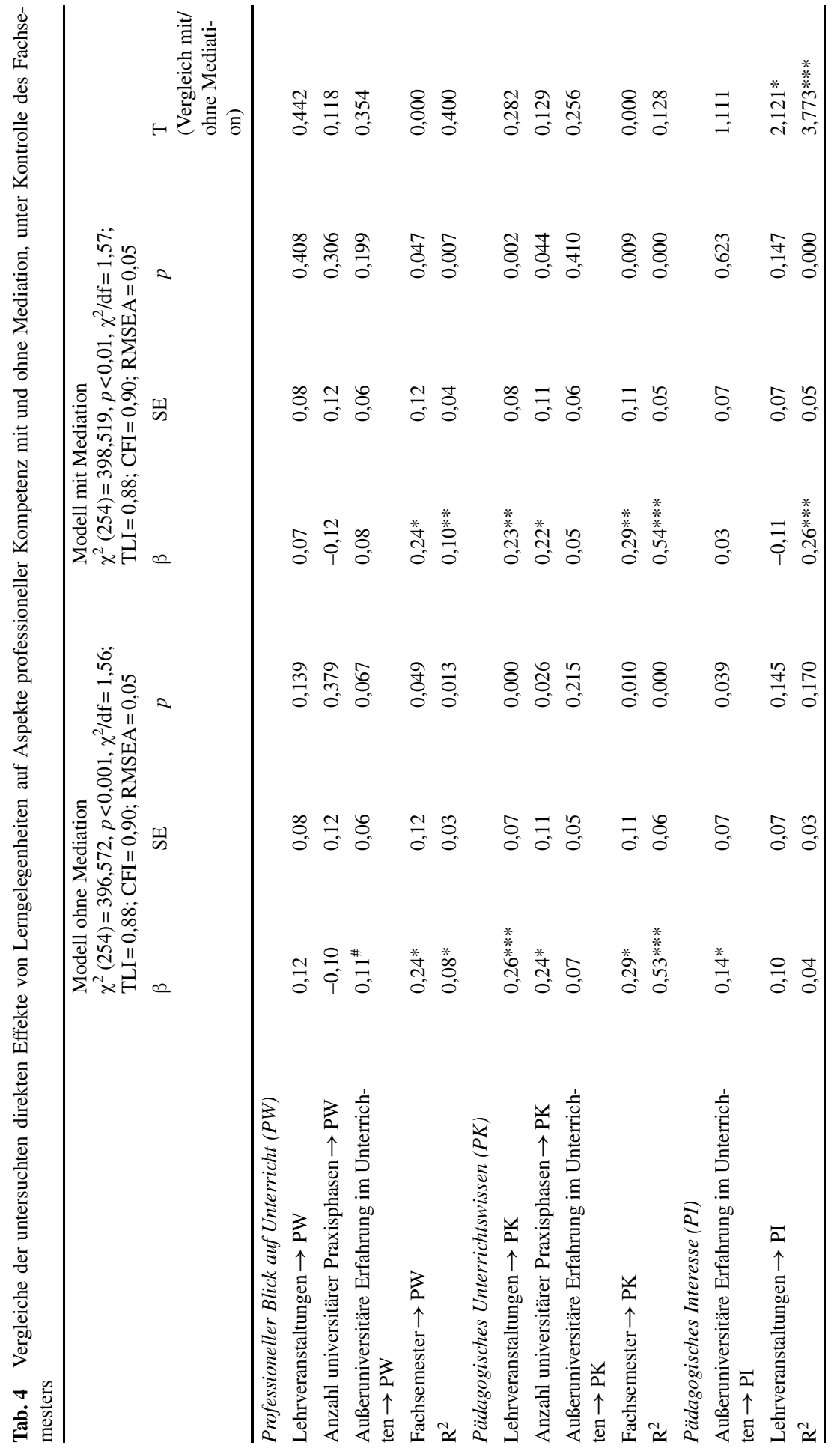




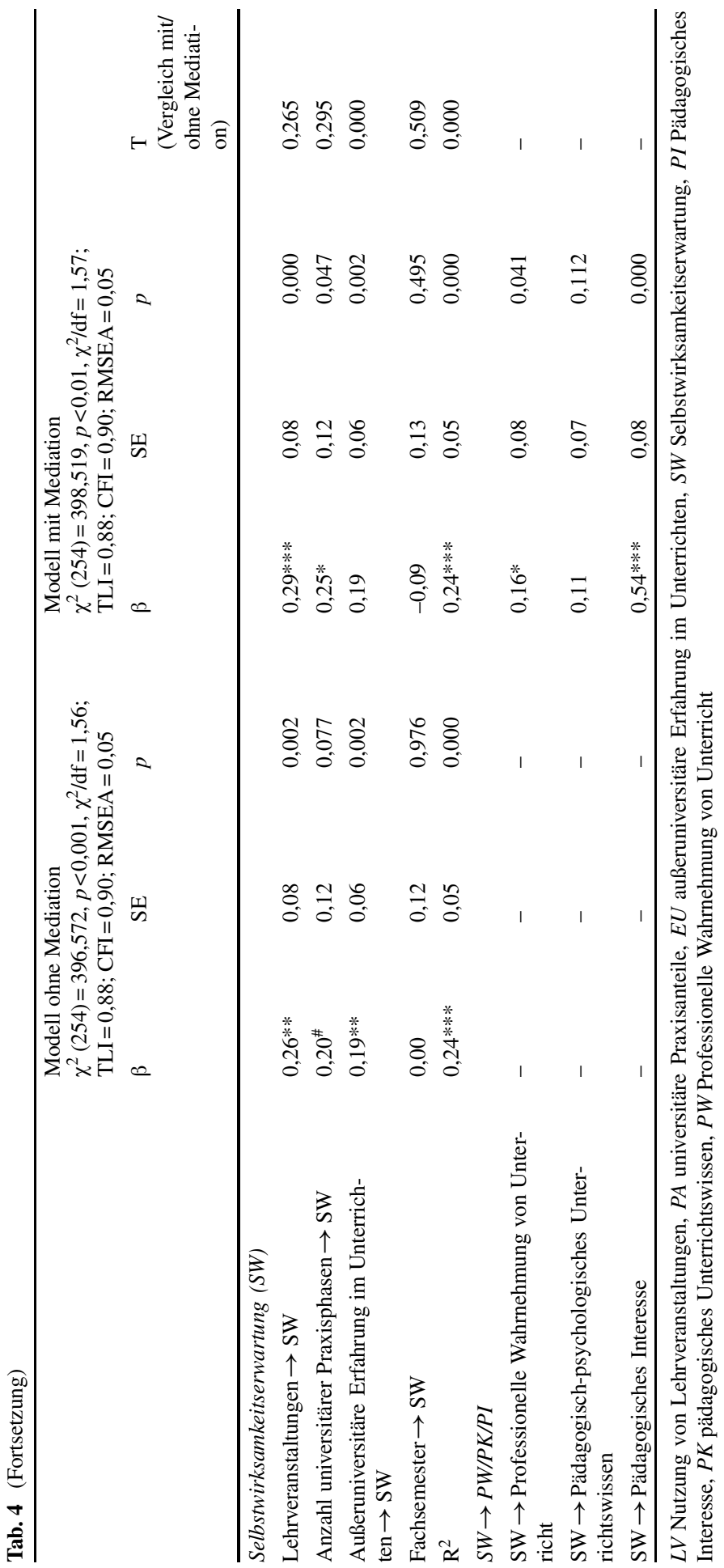


Die pädagogische Vorerfahrung im Unterrichten erklärt nicht das Unterrichtswissen $(\beta=0,02, p=0,158)$ (H6a) und trägt auch nicht indirekt zur professionellen Wahrnehmung der videografierten Unterrichtsstunde bei $(\beta=0,03, p=0,091)$ (H6b). Der direkte Effekt von der außeruniversitären Erfahrung im Unterrichten auf das pädagogische Interesse entfällt und wird vollständig über die Selbstwirksamkeitserwartung mediiert $(\beta=0,10, p=0,006)$ (H6c). Die abgeleiteten Hypothesen für das Mediationsmodell konnten somit teilweise bestätigt werden.

Durch das Einsetzen der Selbstwirksamkeitserwartung als Mediator erhöhen sich die Varianzaufklärungen für das Unterrichtswissen marginal auf 54\% und für die professionelle Wahrnehmung der videografierten Unterrichtsstunde auf $10 \%$. Eine deutliche Verbesserung der Varianzaufklärung wird für das pädagogische Interesse erzielt, das durch das Mediationsmodell nun zu 26\% erklärt werden kann. Ein Vergleich zwischen den untersuchten direkten Effekten mit und ohne Mediation unter Kontrolle des Fachsemesters ist Tab. 4 zu entnehmen.

\section{Diskussion}

\subsection{Interpretation der Ergebnisse}

Die angenommenen Zusammenhänge zwischen Lerngelegenheiten innerhalb und außerhalb des Lehramtsstudiums und den untersuchten Aspekten professioneller Kompetenz konnten nur teilweise bestätigt werden. Im Folgenden werden die Ergebnisse entlang der Hypothesen diskutiert.

\subsubsection{Hypothesen 1a-1c}

Studierende, die angeben, universitäre Lehrveranstaltungen intensiver zu nutzen, verfügen über ein elaborierteres pädagogisches Unterrichtswissen. Dieser direkte Zusammenhang lässt sich vermutlich dadurch erklären, dass sowohl die universitären Lehrveranstaltungen als auch der Wissenstest zum pädagogischen Unterrichtswissen eher deklaratives Wissen ansprechen, welches vor allem im universitären Teil der Lehramtsausbildung vermittelt wird (Kunter und Pohlmann 2009, S. 264). Die professionelle Wahrnehmung der videografierten Unterrichtsstunde, die sich eher dem prozeduralen Wissen (Voss et al. 2014, S. 188) zuordnen lässt, wird nicht durch eine stärkere Nutzung von Lehrveranstaltungen erklärt. Dieses Ergebnis legt zunächst die Vermutung nahe, dass nur dann die Nutzung von Lerngelegenheiten einen positiven Effekt auf das Wissen hat, wenn eine konzeptuelle Passung von Lerngelegenheiten und Wissensformen vorliegt.

Darüber hinaus geben die Studierenden an, sich eher in der Lage zu fühlen, professionellen Unterricht durchzuführen, wenn sie mehr Lehrveranstaltungen im Studium genutzt haben. Eine Erklärung für den positiven Effekt auf die Selbstwirksamkeitserwartung könnte sein, dass diese Studierenden bereits über mehr theoretisches Handwerkszeug für das Unterrichten verfügen, sich deshalb womöglich besser auf die Lehrtätigkeit vorbereitet fühlen und zuversichtlicher im Hinblick auf das eigene Lehren sind. Universitäre Lehrveranstaltungen können darüber hinaus mehrere 
Gelegenheiten für die Entwicklung von Selbstwirksamkeitserleben bereitstellen. In Anlehnung an Bandura (vgl. 1997) kann eine stellvertretende Erfolgserfahrung zum Beispiel das Lesen von Unterrichtsvignetten sein, in denen Lehrkräfte effektiv und professionell mit unterrichtlichen Herausforderungen (z. B. Motivierung von Lernenden) umgehen. Denkbar ist auch, dass Studierende durch eine häufigere Nutzung der Lehrveranstaltungen bereits mehr Feedback von Dozierenden - im Sinne von verbaler Ermutigung - erhalten haben, was wiederum zu einer höheren Selbstwirksamkeitserwartung führt.

Ähnliche Zusammenhänge werden zwischen dem Besuch von Fortbildungen und der Selbstwirksamkeitserwartung von Lehrkräften vermutet (Richter et al. 2013). Es ist jedoch nicht auszuschließen, dass die Wirkrichtung der Effekte zwischen der Selbstwirksamkeitserwartung und der Nutzung von Lerngelegenheiten entgegengesetzt oder wechselseitig ist.

\subsubsection{Hypothesen $2 a-2 c$}

Entgegen der Erwartung trägt die Anzahl absolvierter Praxisphasen nicht dazu bei, dass Studierende mehr relevante Merkmale guten Unterrichts in der Videovignette identifizieren. Dieser Befund widerspricht zunächst der Annahme der konzeptuellen Passung von Lerngelegenheiten und Wissensformen. Eventuell gibt eine genauere Analyse der konkreten Tätigkeiten innerhalb des Praktikums mehr Aufschluss. Zum Beispiel steht die Durchführung pädagogischer Handlungssituationen und die Reflexion dieser Situationen, die vor allem im Praxisblock des Masterstudiums intendiert ist, in einem engen Zusammenhang mit dem prozeduralem Unterrichtswissen (König et al. 2020, S. 79). Und auch die mentorielle Unterstützung in einer Praxisphase stellt eine förderliche Bedingung für einen Zuwachs im handlungsnahen pädagogischen Wissen dar (vgl. König et al. 2020).

Des Weiteren erklärt die Anzahl der Praxisphasen weder die Selbstwirksamkeitserwartung noch das pädagogische Interesse der Studierenden. Da Erfolgserfahrungen und soziale Unterstützung Quellen für die Entwicklung von Selbstwirksamkeitserleben darstellen (Bandura 1997), sollten die Praxisphasen in einer weiteren Analyse auf diese Aspekte hin untersucht werden. Auch Ulrich et al. (vgl. 2020) nennen in ihrem Review die unterstützende Betreuung während des Praktikums als bedeutsames Kriterium für die Entwicklung von Selbstwirksamkeitserleben. Vermutlich haben die universitären Praxisphasen nur dann einen positiven Effekt auf die Selbstwirksamkeitserwartung und das pädagogische Interesse der Studierenden, wenn sie als herausfordernd, aber zu bewältigen wahrgenommen werden.

Studierende, die mehr Praxisphasen im Studium absolviert haben, verfügen entgegen der Erwartung über mehr pädagogisches Unterrichtswissen. Die Ursache für diesen vorher nicht antizipierten Zusammenhang könnte zum einen in der oben diskutierten Konstruktion des Videotests liegen. Zum anderen ist denkbar, dass in den universitären Praxisphasen durch die Reflexion der Praktika mit Mentor*innen oder in praxisbegleitenden Seminaren vor allem das deklarative Wissen über guten Unterricht gestärkt worden ist. 


\subsubsection{Hypothesen 3a-3d}

Im Gegensatz zu den Ergebnisse der LEK-Studie (König und Seifert 2012) trägt die außeruniversitäre Erfahrung im Unterrichten nicht zur Erklärung des pädagogischen Unterrichtswissens bei. Und auch die Anzahl von genannten Qualitätsaspekten im Videotest wird nicht von der außeruniversitären Erfahrung im Unterrichten erklärt. Der Grund für die fehlenden Zusammenhänge könnte darin liegen, dass praktische Erfahrungen, die außerhalb der Universität und vermutlich überwiegend bereits vor Beginn des Lehramtsstudiums gesammelt worden sind, seltener mit theoretischem Wissen verknüpft und reflektiert werden (König et al. 2017; Yost et al. 2000).

Jedoch trägt die außeruniversitäre pädagogische (Vor-)Erfahrung als stärkster Prädiktor signifikant zur Erklärung des pädagogischen Interesses bei. Es ist anzunehmen, dass die Entscheidung für ein Lehramtsstudium vor allem aufgrund positiver Vorerfahrungen in der pädagogischen Arbeit mit Kindern und Jugendlichen getroffen wurde. Je größer die Erfahrung im Unterrichten außerhalb des Lehramtsstudiums ist, desto größer ist auch die Selbstwirksamkeitserwartung der Studierenden. Vermutlich geben Studierende vor allem dann an, über einen längeren Zeitraum außeruniversitär unterrichtet zu haben, wenn sie Erfolgserfahrungen in ihrer Tätigkeit gemacht haben. Diese persönlichen Erfolgserlebnisse führen wiederum zu einer höheren Selbstwirksamkeitserwartung bezogen auf das Unterrichten (Rothland 2015).

Ein weiteres Ergebnis, das sich bereits in den Interkorrelationen angedeutet hat, ist die Relevanz der Selbstwirksamkeitserwartung - sowohl im Hinblick auf Lerngelegenheiten als auch im Hinblick auf die untersuchten Aspekte der professionellen Kompetenz. Setzt man die Selbstwirksamkeitserwartung als Mediator zwischen den Lerngelegenheiten und den Aspekten professioneller Kompetenz ein, erhöht sich die Varianzaufklärung für alle Zielvariablen, insbesondere für das pädagogische Interesse am Lehrberuf.

\subsubsection{Hypothesen $4 a-4 b$}

Entgegen der Erwartung werden Effekte von der Nutzung universitärer Lehrveranstaltungen auf das Unterrichtswissen und auf die professionelle Wahrnehmung der videografierten Unterrichtsstunde nicht über die Selbstwirksamkeitserwartung mediiert. Es scheint also vor allem das erworbene deklarative Wissen aus Seminaren und Vorlesungen zu sein, das direkt mit dem Ergebnis im Wissenstest und im Videotest zusammenhängt.

Jedoch verfügen Studierende, die Lehrveranstaltungen in hohem Maße genutzt haben und sich dadurch selbstwirksamer im Unterrichten fühlen, über ein größeres pädagogisches Interesse. Es ist anzunehmen, dass ein umfangreiches theoretisches Wissen zu mehr Zuversicht führt, effektiv unterrichten zu können. Dies könnte zu einer Verstärkung des pädagogischen Interesses führen, da Studierende das Gefühl haben, die pädagogische Arbeit mit Kindern und Jugendlichen sei für sie machbar und deshalb auch passend. 


\subsubsection{Hypothesen 5a-5c}

Die Ergebnisse für die universitären Praxisphasen fallen ähnlich aus wie für die Nutzung der Lehrveranstaltungen: Es werden keine Mediationseffekte auf das Unterrichtswissen oder die professionelle Wahrnehmung der videografierten Unterrichtsstunde gefunden. Allerdings sind Studierende mit steigender Anzahl absolvierter universitärer Praktika zuversichtlicher, effektiv unterrichten und zu können und geben dadurch ein größeres Interesse an der pädagogischen Arbeit mit Kindern und Jugendlichen an. Die Ursachen für diese Ergebnisse sind vermutlich ähnlich wie für die Effekte von der Nutzung der Lehrveranstaltungen.

\subsubsection{Hypothesen $6 a-6 c$}

Die pädagogische (Vor-)Erfahrung im Unterrichten trägt nicht zur Erklärung des Unterrichtswissens und der professionellen Wahrnehmung von Unterricht bei, auch dann nicht, wenn die Selbstwirksamkeitserwartung als Mediator in das Modell eingesetzt wird. Vermutlich führt die fehlende Theorie-Praxis-Verzahnung in außeruniversitären Lerngelegenheiten dazu, dass diese mit dem untersuchten pädagogischen Unterrichtswissen in keinerlei Art verbunden sind. Der Effekt von der außeruniversitären Erfahrung im Unterrichten auf das pädagogische Interesse der Studierenden wird vollständig über die Selbstwirksamkeitserwartung vermittelt. Zum einen lässt sich dieses Ergebnis darauf zurückführen, dass sowohl die Selbstwirksamkeitserwartung als auch das pädagogische Interesse motivationale Konstrukte sind, die eng miteinander zusammenhängen. Zum anderen ist davon auszugehen, dass Studierende besonders dann ein starkes pädagogisches Interesse an der Arbeit mit Kindern und Jugendlichen haben, wenn sie bereits positive pädagogische (Vor-)Erfahrungen gesammelt haben und deshalb zuversichtlich sind, effektiv unterrichten zu können.

\subsection{Limitationen}

In methodischer Hinsicht sind folgende Limitationen $\mathrm{zu}$ nennen: Auch wenn die Ergebnisse nahelegen, dass die Nutzung von Lerngelegenheiten die Entwicklung professioneller Kompetenz beeinflusst, ist aufgrund der querschnittlichen Untersuchung nicht auszuschließen, dass die Effekte in die entgegengesetzte Richtung wirken oder sich wechselseitig beeinflussen. Es ist zum Beispiel denkbar, dass Studierende, die über eine höhere Selbstwirksamkeitserwartung oder ein höheres pädagogisches Interesse verfügen, vermehrt Lerngelegenheiten nutzen. Daher sollten in längsschnittlichen Analysen Veränderungen von Aspekten professioneller Kompetenz unter Berücksichtigung der Nutzung von Lerngelegenheiten genauer untersucht werden. Eine weitere Limitation, die zu Verzerrungen (Deckeneffekten) im Pfadmodell und zu einer Unterschätzung der Regressionskoeffizienten geführt haben könnte, ist die geringe Variabilität in der Selbstwirksamkeitserwartung und im pädagogischen Interesse bei gleichzeitig hohen Ausprägungen. Zukünftige Studien sollten daher mehr Items einsetzen, die auch zwischen hohen Ausprägungen dieser Kompetenzfacetten differenzieren können. Ferner können fehlende Zusammenhänge zwischen der Anzahl der absolvierten Praxisphasen und den untersuchten Aspek- 
ten der professionellen Kompetenz in der geringen Varianz der Lerngelegenheiten begründet sein. Da es sich bis auf die Ergebnisse in dem Wissens- und Videotest um Selbstaussagen handelt, ist außerdem eine Konfundierung durch geteilte Methodenvarianz nicht auszuschließen. Dies könnte wiederum zu einer Überschätzung der Zusammenhänge geführt haben. Um dieser Problematik zu entgegnen, ist es sinnvoll, die Nutzung von Lerngelegenheiten zusätzlich beispielsweise in Form abgegebener Hausarbeiten, Anwesenheitslisten in Vorlesungen oder Beteiligungsraten in Seminaren zu erheben.

Darüber hinaus stellt die prognostische Validität der eingesetzten Messinstrumente eine weitere mögliche Limitation dar. Inwiefern die außeruniversitäre Erfahrung im Unterrichten ein geeigneter Prädiktor für die Anzahl genannter Qualitätsmerkmale guten Unterrichts im Videotest sein kann, muss kritisch hinterfragt werden, da diese Erfahrungen nicht zwingend vor dem Hintergrund von Aspekten guten Unterrichts (Helmke 2006) reflektiert werden. Außerdem ist fraglich, ob mit dem Videotest die professionelle Wahrnehmung von Unterricht in Form prozeduralen Wissens erhoben wurde. Möglicherweise reicht die alleinige Identifikation von unterrichtsbezogenen Qualitätsmerkmalen (,noticing“, vgl. König et al. 2014; Van Es und Sherin 2008) zur Erhebung des prozeduralen Wissens nicht aus. Der Videotest könnte beispielsweise um die Aufgabe der Interpretation der gesehenen Unterrichtsstunde (,,interpreting“, vgl. König et al. 2014) ergänzt werden. Im letzten Erhebungszeitpunkt der LeveLStudie sollten die Studierenden daher zusätzlich Handlungsalternativen nennen und begründen. Mit dieser Erweiterung sollte das handlungs- und praxisrelevante Wissen genauer gemessen werden. Die Analysen stehen noch aus.

Auf inhaltlicher Ebene ist anzumerken, dass die Lerngelegenheiten im Studium differenzierter erhoben werden sollten. Erst dann kann die angenommene Dichotomie zwischen praxisorientierten vs. theoriebasierten Lerngelegenheiten und dem Zusammenhang mit prozeduralen vs. deklarativen Wissensformen genauer untersucht werden. Eine weitere Schwierigkeit ist in der Generalisierung der berichteten Ergebnisse zu sehen. Die Inhalte des Lehramtsstudiums variieren je nach Universitätsstandort. Dies macht allgemeingültige Aussagen zur Wirksamkeit des Lehramtsstudiums auf die Entwicklung professioneller Kompetenz schwierig. Darüber hinaus sind die eingesetzten Messinstrumente der vorgestellten Untersuchung auf die Bedingungen des Studienstandorts angepasst worden. Daher sind die Ergebnisse nicht unbedingt auf andere Studienstandorte übertragbar.

\section{Implikationen für die Praxis und Ausblick}

Da motivationale Orientierungen einen wichtigen Aspekt der professionellen Kompetenz von Lehrkräften darstellen und positiv mit einer engagierten und effektiven Unterrichtsgestaltung zusammenhängen, liefert die vorgestellte Untersuchung trotz der genannten Einschränkungen wichtige Erkenntnisse.

Die Selbstwirksamkeitserwartung scheint eine zentrale Funktion zwischen Lerngelegenheiten und Aspekten professioneller Kompetenz einzunehmen. Dies gilt insbesondere für das pädagogische Interesse von Lehramtsstudierenden. Die Ergebnisse weisen darauf hin, dass universitäre Lerngelegenheiten so gestaltet werden 
sollten, dass Studierende dort in ausreichendem Maße Erfolgserfahrungen machen können, da diese die Hauptquelle für Selbstwirksamkeitserwartungen darstellen (vgl. Bandura 1997). Darüber hinaus tragen außeruniversitäre (Vor-)Erfahrungen im Unterrichten zu einer höheren Selbstwirksamkeit und einem stärkeren pädagogischen Interesse bei. Auf der Grundlage dieser Befunde ist zum einen denkbar, dass pädagogische Vorerfahrungen als zusätzliches Kriterium zur Vergabe von Studienplätzen im Lehramtsstudium in Betracht gezogen werden sollten. Zum anderen könnten Studierende verstärkt ermutigt werden, neben ihrem Studium vor allem im pädagogischen Bereich tätig zu sein. Hier wären beispielsweise Kooperationen zwischen Universitäten und Schulen bzw. Nachhilfeinstituten denkbar. Diese Maßnahmen könnten dazu beitragen, dass Lehramtsstudierende in höherem Maße intrinsisch motiviert sind und dadurch auch in anderen Bereichen professioneller Kompetenz günstige Entwicklungen zeigen.

Künftige Studien sollten ferner formelle und informelle Lerngelegenheiten differenzierter erfassen. Neben der Untersuchung der quantitativen Nutzung von Lerngelegenheiten könnte die zusätzliche Analyse qualitativer und inhaltlicher Aspekte vielversprechend sein.

In einer Untersuchung von Palmer (vgl. 2007) führten praxisnahe Seminare und Gruppenarbeiten zu einer Erhöhung der Selbstwirksamkeitserwartung. Besonders wichtig für die Aufrechterhaltung der gestärkten Selbstwirksamkeitserwartung war, dass die Studierenden die Möglichkeit hatten, im Anschluss an die Seminare das theoretische und praxisnahe Wissen in Schulklassen anzuwenden. Im Hinblick auf die Qualität von Lerngelegenheiten nennen Stancel-Piątak und Kolleg*innen motivierende Aspekte und direktes Feedback (Stancel-Piątak et al. 2013, S. 191). Des Weiteren konnte gezeigt werden, dass angehende Lehrkräfte, die von ihren Mentor*innen in hohem Maße unterstützt wurden, und die ihren Unterricht zu einem gewissen Grad selbstbestimmt entwickeln durften, über einen professionelleren Blick auf Unterricht und über mehr pädagogisches Wissen verfügen (König et al. 2014, S. 80). Ronfeldt und Reininger konnten zeigen, dass die Zufriedenheit von zukünftigen Lehrkräften beim Unterrichten einen positiven Effekt auf die Selbstwirksamkeitserwartung hatte (vgl. Ronfeldt und Reininger 2012). In der LEK-Studie verfügten Lehramtsstudierende, die Seminare mit Möglichkeiten zur partizipativen Teilnahme besucht hatten, über mehr pädagogisches Unterrichtswissen (König et al. 2012b, S. 258).

Zusätzlich könnte auch die differenziertere Erfassung des pädagogischen Unterrichtswissens aufschlussreich sein. Ähnlich wie in den TEDS-Studien sollten die Items im Wissenstest unterschiedlichen kognitiven Prozessen zugeordnet werden (erinnern, verstehen/analysieren, kreieren, vgl. Blömeke 2010). Diese Vorgehensweise kann beispielsweise zur Klärung der Frage beitragen, ob praxisnahe Lerngelegenheiten stärker mit handlungsorientierten kognitiven Prozessen zusammenhängen.

Darüber hinaus sollten die genannten unterrichtlichen Qualitätsaspekte im Videotest einer detaillierteren qualitativen Analyse unterzogen werden. Sie könnte Aufschluss darüber geben, ob eine bestimmte Sichtweise auf Unterricht eher mit praxisorientierten oder theoriebasierten Lerngelegenheiten zusammenhängt (z. B. stärkerer Fokus auf Unterrichtsmethoden, auf kognitive Aktivierung usw.). Außerdem sollte nicht ausschließlich die Identifikation von unterrichtlichen Qualitätsaspekten ana- 
lysiert werden, sondern darüber hinaus sollten die Nennung und Begründung von Handlungsalternativen als Indikatoren professioneller Unterrichtswahrnehmung untersucht werden. Künftige Analysen werden zeigen, ob universitäre und außeruniversitäre Lerngelegenheiten geeignete Prädiktoren für die professionelle Wahrnehmung von (videografiertem) Unterricht sind.

Um eine Generalisierbarkeit der gefunden Zusammenhänge zu ermöglichen, sollten vergleichbare Untersuchungen auch an anderen Studienstandorten repliziert werden.

Funding Open Access funding enabled and organized by Projekt DEAL.

Open Access Dieser Artikel wird unter der Creative Commons Namensnennung 4.0 International Lizenz veröffentlicht, welche die Nutzung, Vervielfältigung, Bearbeitung, Verbreitung und Wiedergabe in jeglichem Medium und Format erlaubt, sofern Sie den/die ursprünglichen Autor(en) und die Quelle ordnungsgemäß nennen, einen Link zur Creative Commons Lizenz beifügen und angeben, ob Änderungen vorgenommen wurden.

Die in diesem Artikel enthaltenen Bilder und sonstiges Drittmaterial unterliegen ebenfalls der genannten Creative Commons Lizenz, sofern sich aus der Abbildungslegende nichts anderes ergibt. Sofern das betreffende Material nicht unter der genannten Creative Commons Lizenz steht und die betreffende Handlung nicht nach gesetzlichen Vorschriften erlaubt ist, ist für die oben aufgeführten Weiterverwendungen des Materials die Einwilligung des jeweiligen Rechteinhabers einzuholen.

Weitere Details zur Lizenz entnehmen Sie bitte der Lizenzinformation auf http://creativecommons.org/ licenses/by/4.0/deed.de.

\section{Literatur}

Bandura, A. (1997). Self-efficacy. The exercise of control (11. Aufl.). New York: Freeman.

Baumert, J., \& Kunter, M. (2006). Stichwort: Professionelle Kompetenz von Lehrkräften. Zeitschrift für Erziehungswissenschaft, 9(4), 469-520.

Baumert, J., Kunter, M., Blum, W., Brunner, M., Voss, T., Jordan, A., Klusmann, U., Krauss, S., Neubrand, M., \& Tsai, Y.-M. (2010). Teachers' mathematical knowledge, cognitive activation in the classroom, and student progress. American Educational Research Journal, 47(1), 133-180.

Blömeke, S. (Hrsg.). (2008). Professionelle Kompetenz angehender Lehrerinnen und Lehrer. Wissen, Überzeugungen und Lerngelegenheiten deutscher Mathematikstudierender und-referendare; erste Ergebnisse zur Wirksamkeit der Lehrerausbildung. Münster: Waxmann.

Blömeke, S. (Hrsg.). (2010). TEDS-M 2008. Professionelle Kompetenz und Lerngelegenheiten angehender Mathematiklehrkräfte für die Sekundarstufe I im internationalen Vergleich. Münster: Waxmann.

Blömeke, S. (Hrsg.). (2013). Professionelle Kompetenzen im Studienverlauf. Weitere Ergebnisse zur Deutsch-, Englisch- und Mathematiklehrerausbildung aus TEDS-LT. Münster: Waxmann.

Blömeke, S., König, J., Kaiser, G., \& Suhl, U. (2010). Lerngelegenheiten angehender Mathematiklehrkräfte für die Sekundarstufe I im internationalen Vergleich. In S. Blömeke (Hrsg.), TEDS-M 2008. Professionelle Kompetenz und Lerngelegenheiten angehender Mathematiklehrkräfte für die Sekundarstufe I im internationalen Vergleich (S. 97-136). Münster: Waxmann.

Blüthmann, I., Ophardt, D., Thiel, F., \& Felsberger, G. (2011). Wissenserwerb zum Thema Klassenmanagement im Lehramtsstudium. Individuelle und studienbezogene Einflussfaktoren. Unterrichtswissenschaft, 39(4), 290-309.

Bromme, R. (1992). Der Lehrer als Experte. Bern: Huber.

Cicchetti, D. V. (1994). Guidelines, criteria, and rules of thumb for evaluating normed and standardized assessment instruments in psychology. Psychological Assessment, 6(4), 284-290.

Clark, S., \& Newberry, M. (2018). Are we building preservice Teacher self-efficacy? A large-scale study examining Teacher education experiences. Asia-Pacific Journal of Teacher Education, 47(1), 32-47.

Darge, K., Schreiber, M., König, J., \& Seifert, A. (2012). Lerngelegenheiten im erziehungswissenschaftlichen Studium. In J. König \& A. Seifert (Hrsg.), Lehramtsstudierende erwerben pädagogisches Pro- 
fessionswissen. Ergebnisse der Längsschnittstudie LEK zur Wirksamkeit der erziehungswissenschaftlichen Lehrerausbildung (S. 87-118). Münster: Waxmann.

Darling-Hammond, L. (2000a). Teacher quality and student achievement. A review of state policy evidence. Education Policy Analysis Archives, 8(1), 1-44.

Darling-Hammond, L. (2000b). How teacher education matters. Journal of Teacher Education, 51(3), $166-173$.

Van Es, E. A., \& Sherin, M. G. (2008). Mathematics teachers" "learning to notice" in the context of a video club. Teaching and Teacher Education, 24(2), 244-276.

Felbrich, A., Müller, C., \& Blömeke, S. (2008). Lerngelegenheiten in der Lehrerausbildung. In S. Blömeke (Hrsg.), Professionelle Kompetenz angehender Lehrerinnen und Lehrer. Wissen, Überzeugungen und Lerngelegenheiten deutscher Mathematikstudierender und-referendare; erste Ergebnisse zur Wirksamkeit der Lehrerausbildung (S. 327-362). Münster: Waxmann.

Hattie, J. A.C. (2010). The international library of sociology: Visible learning. A synthesis of over 800 meta-analyses relating to achievement. London: Routledge.

Helmke, A. (2006). Was wissen wir über guten Unterricht? Pädagogik, 2(58), 42-45.

Helmke, A. (2010). Schule weiterentwickeln, Unterricht verbessern Orientierungsband: Unterrichtsqualität und Lehrerprofessionalität. Diagnose, Evaluation und Verbesserung des Unterrichts Franz Emanuel Weinert gewidmet (3. Aufl.). Seelze-Velber: Klett.

Holzberger, D., Philipp, A., \& Kunter, M. (2013). How teachers' self-efficacy is related to instructional quality. A longitudinal analysis. Journal of Educational Psychology, 105(3), 774-786.

Holzberger, D., Philipp, A., \& Kunter, M. (2014). Predicting teachers' instructional behaviors. The interplay between self-efficacy and intrinsic needs. Contemporary Educational Psychology, 39(2), $100-111$.

van Iddekinge, C.H., Putka, D.J., \& Campbell, J.P. (2011). Reconsidering vocational interests for personnel selection. The validity of an interest-based selection test in relation to job knowledge, job performance, and continuance intentions. The Journal of applied psychology, 96(1), 13-33.

Kappler, C. (2013). Berufswahlprozesse und Motive angehender Lehrer (1. Aufl.). Bern: Haupt.

Kline, R.B. (2016). Methology in the social sciences: Principles and practice of structural equation modeling (4. Aufl.). London: Taylor \& Francis.

König, J. (2012). Die Entwicklung von pädagogischem Unterrichtswissen: Theoretischer Rahmen, Testinstrument, Skalierung und Ergebnisse. In J. König \& A. Seifert (Hrsg.), Lehramtsstudierende erwerben pädagogisches Professionswissen. Ergebnisse der Längsschnittstudie LEK zur Wirksamkeit der erziehungswissenschaftlichen Lehrerausbildung (S. 141-182). Münster: Waxmann.

König, J., \& Klemenz, S. (2015). Der Erwerb von pädagogischem Wissen bei angehenden Lehrkräften in unterschiedlichen Ausbildungskontexten. Zur Wirksamkeit der Lehrerausbildung in Deutschland und Österreich. Zeitschrift für Erziehungswissenschaft, 18(2), 247-277.

König, J., \& Seifert, A. (Hrsg.). (2012). Lehramtsstudierende erwerben pädagogisches Professionswissen. Ergebnisse der Längsschnittstudie LEK zur Wirksamkeit der erziehungswissenschaftlichen Lehrerausbildung. Münster: Waxmann.

König, J., Tachtsoglou, S., \& Seifert, A. (2012a). Individuelle Voraussetzungen, Lerngelegenheiten und der Erwerb von pädagogischem Professionswissen. In J. König \& A. Seifert (Hrsg.), Lehramtsstudierende erwerben pädagogisches Professionswissen. Ergebnisse der Längsschnittstudie LEK zur Wirksamkeit der erziehungswissenschaftlichen Lehrerausbildung (S. 234-283). Münster: Waxmann.

König, J., Kaiser, G., \& Felbrich, A. (2012b). Spiegelt sich pädagogisches Wissen in den Kompetenzselbsteinschätzungen angehender Lehrkräfte? Zum Zusammenhang von Wissen und Überzeugungen am Ende der Lehrerausbildung. Zeitschrift für Pädagogik, 58(4), 476-491.

König, J., Blömeke, S., Klein, P., Suhl, U., Busse, A., \& Kaiser, G. (2014). Is teachers' general pedagogical knowledge a premise for noticing and interpreting classroom situations? A video-based assessment approach. Teaching and Teacher Education, 38, 76-88.

König, J., Rothland, M., Tachtsoglou, S., \& Klemenz, S. (2016). Comparing the change of teaching motivations among preservice teachers in Austria, Germany, and Switzerland. Do in-school learning opportunities matter? International Journal of Higher Education, 5(3). https://doi.org/10.5430/ijhe. v5n3p91.

König, J., Ligtvoet, R., Klemenz, S., \& Rothland, M. (2017). Effects of opportunities to learn in teacher preparation on future teachers? General pedagogical knowledge: analyzing program characteristics and outcomes. Studies in Educational Evaluation, 53, 122-133.

König, J., Darge, K., \& Kramer, C. (2020). Kompetenzentwicklung im Praxissemester: Zur Bedeutung schulpraktischer Lerngelegenheiten auf den Erwerb von pädagogischem Wissen bei Lehramtsstudie- 
renden. In I. Ulrich \& A. Gröschner (Hrsg.), Praxissemester im Lehramtsstudium in Deutschland. Wirkungen auf Studierende (S. 67-95). Wiesbaden: Springer.

Krapp, A., \& Ryan, R. M. (2002). Selbstwirksamkeit und Lernmotivation. Eine kritische Betrachtung der Theorie von Bandura aus der Sicht der Selbstbestimmungstheorie und der pädagogisch-psychologischen Interessentheorie. In M. Jerusalem \& D. Hopf (Hrsg.), Selbstwirksamkeit und Motivationsprozesse in Bildungsinstitutionen (Zeitschrift für Pädagogik, 44. Beiheft, S. 54-82). Weinheim: Beltz.

Kunina-Habenicht, O., Schulze-Stocker, F., Kunter, M., Baumert, J., Leutner, D., Förster, D., Lohse-Bossenz, H., \& Terhart, E. (2013). Die Bedeutung der Lerngelegenheiten im Lehramtsstudium und deren individuelle Nutzung für den Aufbau des bildungswissenschaftlichen Wissens. Zeitschrift für Pädagogik, 59(1), 1-23.

Kunter, M., \& Pohlmann, B. (2009). Lehrer. In E. Wild \& J. Möller (Hrsg.), Pädagogische Psychologie (Lehrbuch mit Online-Materialien, S. 261-282). Dordrecht: Springer.

Kunter, M., Kleickmann, T., Klusmann, U., \& Richter, D. (2011). Die Entwicklung professioneller Kompetenz von Lehrkräften. In M. Kunter, J. Baumert, W. Blum, U. Klusmann, S. Krauss \& M. Neubrand (Hrsg.), Professionelle Kompetenz von Lehrkräften. Ergebnisse des Forschungsprogramms COACTIV (S. 55-68). Münster, München: Waxmann.

Lerche, T., Weiß, S., \& Kiel, E. (2013). Mythos pädagogische Vorerfahrung. Zeitschrift für Pädagogik, 59(5), 762-782.

Little, T. D., Cunningham, W. A., Shahar, G., \& Widaman, K. F. (2002). To parcel or not to parcel. Exploring the question, weighing the merits. Structural Equation Modeling: A Multidisciplinary Journal, 9(2), 151-173.

MacKinnon, D.P. (2008). Introduction to Statistical Mediation Analysis (1st ed.). Routledge. https://doi. org/10.4324/9780203809556.

Müller, K. (2010). Das Praxisjahr in der Lehrerbildung. Empirische Befunde zur Wirksamkeit studienintegrierter Langzeitpraktika. Bad Heilbrunn: Klinkhardt.

Palmer, D. (2007). Durability of changes in self-efficacy of preservice primary teachers. International Journal of Science Education, 28(6), 655-671.

Paulick, I., Retelsdorf, J., \& Möller, J. (2013). Motivation for choosing teacher education. Associations with teachers' achievement goals and instructional practices. International Journal of Educational Research, 61, 60-70.

Pohlmann, B., \& Möller, J. (2010). Fragebogen zur Erfassung der Motivation für die Wahl des Lehramtsstudiums (FEMOLA)1. Zeitschrift für Pädagogische Psychologie, 24(1), 73-84.

Richter, D., Engelbert, M., Weirich, S., \& Pant, A. H. (2013). Differentielle Teilnahme an Lehrerfortbildungen und deren Zusammenhang mit professionsbezogenen Merkmalen von Lehrkräften. Zeitschrift für Pädagogische Psychologie, 27(3), 193-207.

Ronfeldt, M., \& Reininger, M. (2012). More or better student teaching? Teaching and Teacher Education, 28(8), 1091-1106.

Rothland, M. (2015). Die Bedeutung pädagogischer (Vor-)Erfahrungen von Lehramtsstudierenden - ein Mythos. Zeitschrift für Pädagogik, 61(2), 270-281.

Schiefele, U., \& Urhahne, D. (2000). Motivationale und volitionale Bedingungen der Studienleistung. In U. Schiefele \& K.-P. Wild (Hrsg.), Interesse und Lernmotivation. Neue Studien zu Entwicklung und Wirkungen (S. 183-206). Münster: Waxmann.

Schiefele, U., \& Wild, K.-P. (Hrsg.). (2000). Interesse und Lernmotivation. Neue Studien zu Entwicklung und Wirkungen. Münster: Waxmann.

Schiefele, U., Köller, O., \& Schaffner, E. (2018). Intrinsische und extrinsische Motivation. In D. H. Rost, J. R. Sparfeldt \& S. Buch (Hrsg.), Beltz Psychologie 2018: Handwörterbuch pädagogische Psychologie (5. Aufl., S. 309-319). Weinheim: Beltz.

Schreiber, M., Darge, K., Tachtsoglou, S., König, J., \& Rothland, M. (2012a). EMW - Entwicklung von berufsspezifischer Motivation und pädagogischem Wissen in der Lehrerausbildung (Codebook zum Fragebogen Messzeitpunkt 1, Teil 1, DE/AT/CH).

Schreiber, M., Darge, K., König, J., \& Seifert, A. (2012b). Individuelle Voraussetzungen von zukünftigen Lehrkräften. In J. König \& A. Seifert (Hrsg.), Lehramtsstudierende erwerben pädagogisches Professionswissen. Ergebnisse der Längsschnittstudie LEK zur Wirksamkeit der erziehungswissenschaftlichen Lehrerausbildung (S. 119-140). Münster: Waxmann.

Schulte, K. (2008). Selbstwirksamkeitserwartungen in der Lehrerbildung. Zur Struktur und dem Zusammenhang von Lehrer-Selbstwirksamkeitserwartungen, Pädagogischem Professionswissen und Persönlichkeitseigenschaften bei Lehramtsstudierenden und Lehrkräften (Dissertationsschrift). Göttingen. http://hdl.handle.net/11858/00-1735-0000-0006-AD1A-3. 
Schulte, K., Bögeholz, S., \& Watermann, R. (2008). Selbstwirksamkeitserwartungen und Pädagogisches Professionswissen im Verlauf des Lehramtsstudiums. Zeitschrift für Erziehungswissenschaft, 11(2), 268-287.

Schulze-Stocker, F., Holzberger, D., Kunina-Habenicht, O., Terhart, E., \& Kunter, M. (2016). Spielen Studienschwerpunkte wirklich eine Rolle? Zeitschrift für Erziehungswissenschaft, 19(3), 599-623.

Schwarzer, R., \& Jerusalem, M. (1999). Skalen zur Erfassung von Lehrer- und Schülermerkmalen. Dokumentation der psychometrischen Verfahren im Rahmen der Wissenschaftlichen Begleitung des Modellversuchs Selbstwirksame Schulen. Berlin: R. Schwarzer.

Shulman, L. (1987). Knowledge and teaching. Foundations of the new reform. Harvard Educational Review, 57(1), 1-23.

Stancel-Piątak, A., Buchholtz, C., \& Schwippert, K. (2013). Anlage und Design der Studie TEDS-LT. In S. Blömeke (Hrsg.), Professionelle Kompetenzen im Studienverlauf. Weitere Ergebnisse zur Deutsch-, Englisch- und Mathematiklehrerausbildung aus TEDS-LT (S. 25-46). Münster: Waxmann.

Tachtsoglou, S., \& König, J. (2018). Der Einfluss von Lerngelegenheiten in der Lehrerausbildung auf das pädagogische Wissen angehender Englischlehrkräfte. Journal für Bildungsforschung Online, 10(2), 3-33.

Terhart, E., Schulze-Stocker, F., Kunina-Habenicht, O., Dicke, T., Förster, D., Lohse-Bossenz, H., Gößling, J., Kunter, M., Baumert, J., \& Leutner, D. (2012). Bildungswissenschaftliches Wissen und der Erwerb professioneller Kompetenz in der Lehramtsausbildung. Eine Kurzdarstellung des BilWiss-Projekts. Lehrerbildung auf dem Prüfstand, 5(1), 96-106.

Tschannen-Moran, M., \& Hoy, A.W. (2007). The differential antecedents of self-efficacy beliefs of novice and experienced teachers. Teaching and Teacher Education, 23(6), 944-956.

Tschannen-Moran, M., Hoy, A. W., \& Hoy, W. K. (1998). Teacher efficacy. Its meaning and measure. Review of Educational Research, 68(2), 202-248.

Ulrich, I., Klingebiel, F., Bartels, A., Staab, R., Scherer, S., \& Gröschner, A. (2020). Wie wirkt das Praxissemester im Lehramtsstudium auf Studierende? Ein systematischer Review. In I. Ulrich \& A. Gröschner (Hrsg.), Praxissemester im Lehramtsstudium in Deutschland. Wirkungen auf Studierende (S. 1-66). Wiesbaden: Springer.

Voss, T., Kunter, M., Seiz, J., Hoehne, V., \& Baumert, J. (2014). Die Bedeutung des pädagogisch-psychologischen Wissens von angehenden Lehrkräften für die Unterrichtsqualität. Zeitschrift für Pädagogik, 60(2), 184-201.

Voss, T., Kunina-Habenicht, O., Hoehne, V., \& Kunter, M. (2015). Stichwort Pädagogisches Wissen von Lehrkräften. Empirische Zugänge und Befunde. Zeitschrift für Erziehungswissenschaft, 18(2), 187-223.

Weinstein, C. S. (1989). Teacher education students' preconceptions of teaching. Journal of Teacher Education, 40(2), 53-60.

Yost, D. S., Sentner, S. M., \& Forlenza-Bailey, A. (2000). An Examination of the Construct of Critical Reflection: Implications for Teacher Education Programming in the 21st Century. Journal of Teacher Education, 51(1), 39-49. 